\title{
Syntheses of Several Dihydrochalcone-Amino Acid and -Peptide Conjugates
}

\author{
Shintaro KamIYA, ${ }^{*}$ Sachiko ESAKI, ${ }^{* *}$ Naoko SHIBA* \\ and Takeshi HoSHI ${ }^{* * *}$ \\ * Laboratory of Organic Chemistry, ${ }^{* *}$ Laboratory of Food Chemistry \\ and ${ }^{* * *}$ Laboratory of Physiology, \\ School of Food and Nutritional Sciences, University of Shizuoka, \\ Yada 395, Shizuoka 422, Japan \\ Received December 12, 1988
}

\begin{abstract}
In order to find out some specific blockers of the membrane transport of amino acids and small peptides in animal small intestine and kidney, the following dihydrochalcone (DHC)-amino acid and -peptide conjugates were synthesized. [ $N$-[(6'-hydroxy-4,4'-dimethoxy-DHC)-2'-yloxy]acetylglycyl]-

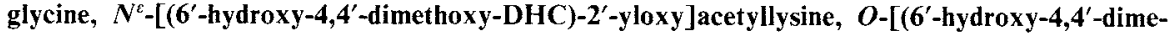
thoxy-DHC)-2'-yl $]$ homoserine, $\left[O-\left[\left(6^{\prime}\right.\right.\right.$-hydroxy-4,4'-dimethoxy-DHC)-2'-yl $]$ homoseryl $]$ glycine, $N$ glycyl-O- $O\left(6^{\prime}\right.$-hydroxy-4,4'-dimethoxy-DHC)-2'-yl]homoserine, and $\left[O-\left[\left(4,4^{\prime}, 6^{\prime}\right.\right.\right.$-trihydroxy-DHC)$2^{\prime}$-yl] homoseryl]glycine.
\end{abstract}

Among the naturally occurring dihydrochalcone (DHC) derivatives, phlorizin ${ }^{1)}$ is produced in most species of the genus Malus and has the structure of $2^{\prime}-(\beta$-D-glucopyranosyloxy)-4,4',6'-trihydroxy-DHC (1). Compound $\mathbf{1}$ is known as a powerful inhibitor ${ }^{2)}$ of $\mathrm{Na}^{+}$. dependent $\mathrm{D}$-glucose uptake in the small intestine and kidney of animals. Its inhibition constant $(K i)$ is usually 1000 times lower than the $\mathrm{Km}$ value of $\mathrm{D}$-glucose, and the inhibition is purely competitive. ${ }^{3)}$ Phloretin (2), the aglycone of $\mathbf{1}$, is much less potent than $\mathbf{1}$ in its ability to block $\mathrm{Na}^{+}$-dependent $\mathrm{D}$-glucose transport, and the mode of its action is noncompetitive. It has generally been considered that the sugar carrier has two separate binding sites, the "sugar" and "phenol" sites, and that phlorizin binds strongly to the carrier by interacting with the two sites. $\left.{ }^{4}\right)$

At the present time, the active transport of such organic solutes as glucose, amino acids and peptides across the intestinal and renal epithelia is known to be mediated by respective carriers (protein) located in the brush border membranes. The cation dependence of these carriers is different in each. D-Glucose carrier is $\mathrm{Na}^{+}$-dependent in the presence of $\mathrm{Na}^{+}$; however, it becomes $\mathrm{H}^{+}$-dependent when $\mathrm{Na}^{+}$ is absent. ${ }^{5)}$ Phlorizin competitively inhibits both $\mathrm{Na}^{+}$-dependent and $\mathrm{H}^{+}$-dependent $\mathrm{D}$ glucose transport. The carrier for neutral amino acids is completely $\mathrm{Na}^{+}$-dependent, $\left.{ }^{6}\right)$ and that for small peptides ( $\mathrm{di}-$ and tripeptides) is entirely dependent on the $\mathrm{H}^{+}$gradient. ${ }^{6,7)}$ Phloretin was found to partially inhibit both $\mathrm{Na}^{+}$-dependent amino acid and $\mathrm{H}^{+}$-dependent peptide transport, as it does for the $\mathrm{Na}^{+}\left(\mathrm{H}^{+}\right)$-dependent sugar transport (Hoshi, unpublished observations).

From an analogy with the biological function of 1 , it may be expected that, among the phloretin-amino acid or peptide conjugates, there must be some which possess the properties of interacting with amino acid and peptide carriers, respectively, to inactivate their transport action. If such blockers can be found, they will be powerful tools for an extensive elucidation of the transport mechanisms and the significance of amino acid and small peptide carriers in the small intestine.

For the foregoing purpose, the present study was designed to synthesize the titled compounds.

The catalytic hydrogenation of 5-hydroxy- 
$4^{\prime}, 7$-dimethoxyflavanone ${ }^{8)}(3)$ over $\mathrm{Pd}-\mathrm{C}$ was markedly accelerated by the addition of small amounts of triethylamine to afford $2^{\prime}, 6^{\prime}$-dihydroxy-4,4'-dimethoxy-DHC (4) in good yields. Condensation of $\mathbf{4}$ with tetra- $O$-acetyl$\alpha$-D-glucopyranosyl bromide (5) in the presence of silver carbonate and 4A molecular sieves gave a coupling product, which, by treating with methanolic sodium methoxide, provided $\quad 2^{\prime}-\left(\beta\right.$-D-glucopyranosyloxy)- $6^{\prime}$-hydroxy-4,4'-dimethoxy-DHC (6) in a $36.6 \%$ overall yield based on 4 . The anomeric configuration of the glycosidic linkage was deduced to be $\beta$-form on the basis of Klyne's rule $^{9)}$ and the NMR spectrum $\left(J_{1^{\prime}, 2^{\prime}}=8 \mathrm{~Hz}\right)$.

The inhibitory activity of 6 for the absorption of glucose into the small intestine was almost the same as that of $\mathbf{1}$ (Hoshi, unpublished data), showing that the replacement of two hydroxyl groups at the C-4 and C-4' positions of the aglycone moiety of $\mathbf{1}$ by a methoxy group did not influence the activity of 1. Accordingly, we first attempted to synthesize the 4,4'-dimethylphoretin-amino acid and -peptide conjugates.

The reaction of 4 with ethyl monochloroacetate yielded 2'-(ethoxycarbonyl)methoxy6'-hydroxy-4,4'-dimethoxy-DHC (7), which, after alkaline hydrolysis, afforded $2^{\prime}$-(carboxy)methoxy-6'-hydroxy-4,4'-dimethoxyDHC (8) in a $47.5 \%$ yield. Coupling 8 with glycylglycine ethyl ester hydrochloride (9) in DMF, using diethylphosphorocyanidate $(\mathrm{DEPC})^{10)}$ as a condensing agent, afforded a $75 \%$ yield of $\left[N-\left[\left(6^{\prime}\right.\right.\right.$-hydroxy-4, $4^{\prime}$-dimethoxy-DHC)-2'yloxy]acetylglycyl]glycine ethyl ester (10). After alkaline hydrolysis, 10 gave $\left[N-\left[\left(6^{\prime}\right.\right.\right.$-hydroxy-4,4'-dimethoxy-DHC)-2' yloxy]acetylglycyl]glycine (11) in a quantitative yield. Similarly, 8 was treated with $N^{\alpha}$ benzyloxycarbonyl-L-lysine benzyl ester benzenesulfonate (12) in the presence of DEPC and triethylamine in DMF to furnish $N^{\alpha}$ benzyloxycarbonyl- $N^{\varepsilon}$-[(6 $6^{\prime}$-hydroxy $-4,4^{\prime}$-dimethoxy-DHC)-2'-yloxy]-acetyl-L-lysine benzyl ester (13) in a $48.3 \%$ yield. Hydrogenolysis of 13 over $\mathrm{Pd}-\mathrm{C}$ in acetic acid provided $N^{\varepsilon}$-[6 $6^{\prime}$-hydroxy-4,4' -dimethoxy-DHC)-2'

yloxy]acetyl-L-lysine (14) in a $70.3 \%$ yield.

Condensation of 4 with 2-[(benzyloxycarbonyl)amino]-4-bromobutanoic acid methyl ester $(15)^{11)}$ was carried out in the presence of potassium carbonate in DMF to give $N$ benzyloxycarbonyl- $O-\left[\left(6^{\prime}-\right.\right.$ hydroxy- $4,4 \rightarrow-\mathrm{di}^{-}$ methoxy-DHC)-2'-yl]homoserine methyl ester (16) in a $60.9 \%$ yield. Compound 16 underwent hydrolysis to $\mathrm{N}$-benzyloxycarbonyl- $O$ [(6'-hydroxy-4,4'-dimethoxy-DHC $]-2^{\prime}$-yl $]$ homoserine (17) in a yield of $92.3 \%$ after treating with aqueous sodium hydroxide. Hydrogenolytic deprotection of $\mathbf{1 7}$ afforded $O$ [(6'-hydroxy-4,4'-dimethoxy-DHC)-2'-yl]homoserine (18). Compound 17 was coupled with glycine benzyl ester tosylate (19) in the presence of DEPC and triethylamine in $\mathrm{DMF}$ to give $\left[N\right.$-benzyloxycarbonyl- $O-\left[\left(6^{\prime}\right.\right.$-hydroxy-4,4'-dimethoxy-DHC)-2'-yl]homoseryl]glycine benzyl ester (20) in an $82.6 \%$ yield. The treatment of $\mathbf{2 0}$ with hydrogen over $\mathrm{Pd}-\mathrm{C}$ resulted in the formation of $\left[O-\left[\left(6^{\prime}\right.\right.\right.$-hydroxy4,4'-dimethoxy-DHC)-2'-yl]homoseryl]glycine (21) in a $98.5 \%$ yield.

As an alternative synthetic method for 21, 17 was treated with glycine ethyl ester hydro-

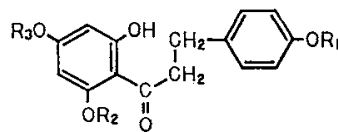

28. $\mathrm{R}_{1}=\mathrm{R}_{3}=\mathrm{H}$ $\mathrm{R}_{2}=\mathrm{CH}_{2} \mathrm{CH}_{2} \mathrm{CHCONHCH} \mathrm{CO}_{2} \mathrm{H}$ $\mathrm{NH}_{2} \cdot \mathrm{HCl}$
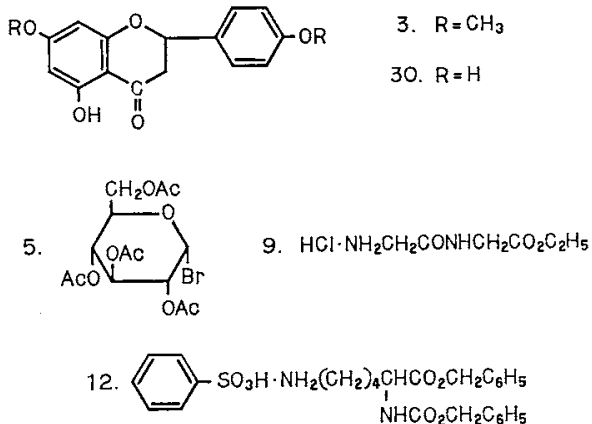

15. $\mathrm{BrCH}_{2} \mathrm{CH}_{2} \mathrm{GHCO}_{2} \mathrm{CH}_{3}$ $\mathrm{NHCO}_{2} \mathrm{CH}_{2} \mathrm{C}_{6} \mathrm{H}_{5}$ 19. $\mathrm{CH}_{3}-\mathrm{SO}_{3} \mathrm{H} \cdot \mathrm{NH}_{2} \mathrm{CH}_{2} \mathrm{CO}_{2} \mathrm{CH}_{2} \mathrm{C}_{6} \mathrm{H}_{5}$

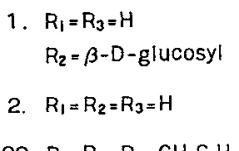

29. $\mathrm{R}_{1}=\mathrm{R}_{2}=\mathrm{R}_{3}=\mathrm{CH}_{2} \mathrm{C}_{6} \mathrm{H}_{5}$

3. $\mathrm{R}=\mathrm{CH}_{3}$ 30. $\mathrm{R}=\mathrm{H}$ $\mathrm{NHCO}_{2} \mathrm{CH}_{2} \mathrm{C}_{6} \mathrm{H}_{5}$

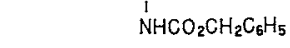




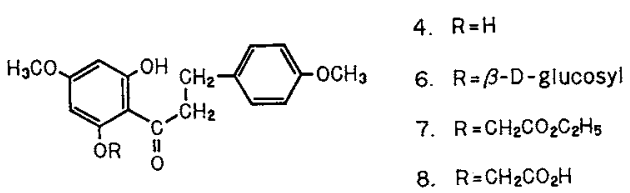

10. $\mathrm{R}=\mathrm{CH}_{2} \mathrm{CONHCH}_{2} \mathrm{CONHCH}_{2} \mathrm{CO}_{2} \mathrm{C}_{2} \mathrm{H}_{5}$

11. $\mathrm{R}=\mathrm{CH}_{2} \mathrm{CONHCH}_{2} \mathrm{CONHCH}_{2} \mathrm{CO}_{2} \mathrm{H}$

13. $\mathrm{R}=\mathrm{CH}_{2} \mathrm{CONH}\left(\mathrm{CH}_{2}\right)_{4} \mathrm{CHCO}_{2} \mathrm{CH}_{2} \mathrm{C}_{6} \mathrm{H}_{5}$ $\mathrm{NHCO}_{2} \mathrm{CH}_{2} \mathrm{C}_{6} \mathrm{H}_{5}$

14. $\mathrm{R}=\mathrm{CH}_{2} \mathrm{CONH}\left(\mathrm{CH}_{2}\right)_{4} \mathrm{CHCO}_{2} \mathrm{H}$

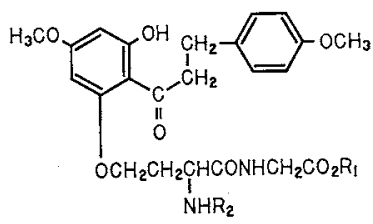

20. $\mathrm{R}_{1}=\mathrm{CH}_{2} \mathrm{C}_{6} \mathrm{H}_{5}$ $\mathrm{R}_{2}=\mathrm{CO}_{2} \mathrm{CH}_{2} \mathrm{C}_{6} \mathrm{H}_{5}$

21. $R_{1}=R_{2}=H$

$$
\text { 22. } \begin{aligned}
\mathrm{R}_{1} & =\mathrm{C}_{2} \mathrm{H}_{5} \\
\mathrm{R}_{2} & =\mathrm{CO}_{2} \mathrm{CH}_{2} \mathrm{C}_{6} \mathrm{H}_{5} \\
\text { 23. } & \mathrm{R}_{\mathrm{t}}=\mathrm{H} \\
\mathrm{R}_{2} & =\mathrm{CO}_{2} \mathrm{CH}_{2} \mathrm{C}_{6} \mathrm{H}_{5}
\end{aligned}
$$

chloride by a method similar to that already described to afford [ $N$-benzyloxycarbonyl- $O$ $\left[\left(6^{\prime}\right.\right.$-hydroxy-4,4'-dimethoxy-DHC)-2'-yl]homoseryl]glycine ethyl ester (22). After alkaline hydrolysis, 22 gave [ $N$-benzyloxycarbonyl$O$-[(6'-hydroxy-4,4'-dimethoxy-DHC)-2'-yl]homoseryl]glycine (23), which was subsequently deprotected with hydrogen to yield 21 in a $43.9 \%$ overall yield based on 22 .

Hydrogenolysis of $\mathbf{1 6}$ in the presence of an equimolar amount of hydrochloric acid in dioxane afforded $O$-[(6'-hydroxy-4,4'-dimethoxy-DHC)-2'-yl]homoserine methyl ester hydrochloride (24) in an $81.5 \%$ yield. The treatment of $\mathbf{2 4}$ with $\mathrm{N}$-benzyloxycarbonylglycine in DMF using DEPC as a catalyst resulted in the formation of $N$-[(benzyloxycarbonyl)glycyl $]-O$-[(6'-hydroxy-4,4'-dimethoxy-DHC)-2'yl]homoserine methyl ester (25) in a $67.9 \%$ yield. Alkaline hydrolysis of $\mathbf{2 5}$ proceeded smoothly to yield $N$-[(benzyloxycarbonyl)glycyl]- $O$-[(6'-hydroxy-4,4'-dimethoxy-DHC)-2'yl]homoserine (26) in a quantitative yield. Hydrogenolytic deprotection of $\mathbf{2 6}$ gave $N$ glycyl-O-[(6'-hydroxy-4,4'-dimethoxy-DHC)$2^{\prime}$-yl]homoserine (27) in a $69.9 \%$ yield.
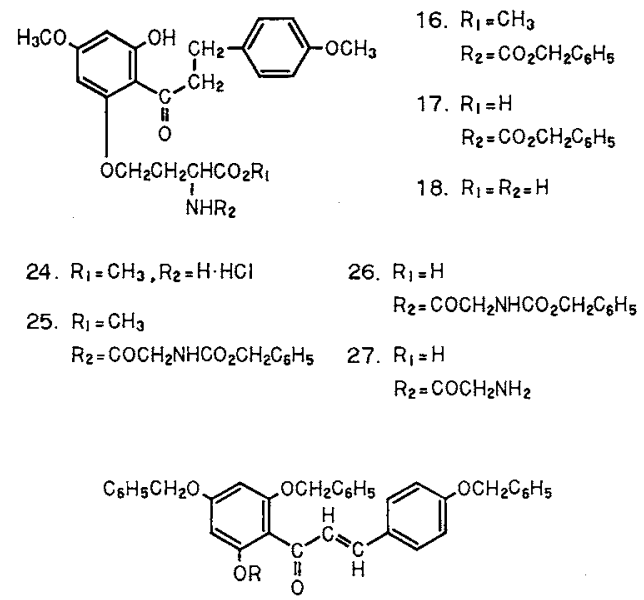

31. $\mathrm{R}=\mathrm{H}$ 32. $\mathrm{R}=\underset{\mathrm{CH}}{\stackrel{\mathrm{N}}{\mathrm{N}} \mathrm{CHCO}_{2} \mathrm{CH}_{2} \mathrm{CH}_{6} \mathrm{H}_{5}}$

33. $\mathrm{R}=\mathrm{CH}_{2} \mathrm{CH}_{2} \mathrm{CHCO}_{2} \mathrm{H}$

$\mathrm{NHCO}_{2} \mathrm{CH}_{2} \mathrm{C}_{6} \mathrm{H}_{5}$

34. $\mathrm{R}=\mathrm{CH}_{2} \mathrm{CH}_{2} \mathrm{CHCONHCH} \mathrm{CH}_{2} \mathrm{CH}_{2} \mathrm{C}_{6} \mathrm{H}_{5}$

$\mathrm{NHCO}_{2} \mathrm{CH}_{2} \mathrm{O}_{5} \mathrm{H}_{5}$

To prepare $\left[O-\left[\left(4,4^{\prime}, 6^{\prime}\right.\right.\right.$-trihydroxy-DHC)2'-yl]homoseryl]glycine hydrochloride (28), direct benzylation of $\mathbf{2}$ with three molar equivalents of benzyl bromide in the presence of potassium carbonate in DMF was examined. However, the desired 4,4',6'-tribenzyloxy-2'hydroxy-DHC (29) was obtained in a poor yield, together with many unidentified byproducts. As the yield of $\mathbf{2 9}$ was not improved by varying the conditions, we finally adopted the benzylation of 4',5,7-trihydroxyflavanone (naringenin, 30) with three molar equivalents of benzyl bromide as already described, and 4,4',6'-tribenzyloxy-2'-hydroxychalcone (31) could be obtained in a good yield.

Treatment of $\mathbf{3 1}$ with $\mathbf{1 5}$ in acetone in the presence of potassium carbonate for $48 \mathrm{hr}$ under reflux, following by chromatographic purification on a silica gel column, yielded $N$ benzyloxycarbonyl-O-[(4,4',6'-tribenzyloxychalcone)-2'-yl]homoserine methyl ester (32), which was subsequently hydrolyzed with sodium hydroxide in aqueous acetone to give $\mathrm{N}$ benzyloxycarbonyl-O-[(4,4',6'-tribenzyloxychalcone)-2'-yl]homoserine (33). Coupling of 33 with 19 in the presence of DEPC and 
triethylamine in DMF resulted in the formation of $[N$-benzyloxycarbonyl-O-[(4,4',6'-tribenzyloxychalcone)-2'-yl]homoseryl]glycine benzyl ester $(34,85.8 \%)$, which was hydrogenolyzed to give $\mathbf{2 8}$ in an $87 \%$ yield.

The results of a biological investigation of the synthetic compounds $(\mathbf{1 1}, \mathbf{1 4}, \mathbf{2 1}, 27$ and 28) will be reported by one of the authors (Hoshi) elsewhere.

\section{Experimental}

Thin-layer chromatography (TLC) was performed on Silica Gel G (Merck), with detection by charring with sulfuric acid, or by spraying with $2 \%$ ferric chloride in ethanol or with $1 \%$ ninhydrin in butanol. Column chromatography was carried out on Kieselgel G 60 , and optical rotations were measured with a Horiba SEPA-200 digital polarimeter. NMR spectra (internal tetramethylsilane) were recorded with a Hitachi R-90 H spectrometer, and UV spectra were measured with a Hitachi double-wavelength (557) spectrophotometer.

$2^{\prime}, 6^{\prime}$-Dihydroxy-4,4'-dimethoxydihydrochalcone (4). To a solution of $3(3 \mathrm{~g})$ in ethyl acetate $(130 \mathrm{ml})$ were added $10 \% \mathrm{Pd}-\mathrm{C}(2 \mathrm{~g})$ and triethylamine $(1.5 \mathrm{ml})$, before the mixture was stirred under hydrogen for $4 \mathrm{hr}$ at room temperature. After filtration, the filtrate was evaporated and a residue was crystallized from chloroform-ethanol to give $4(2.6 \mathrm{~g}, 86.1 \%)$ as colorless needles, $\mathrm{mp} 142^{\circ} \mathrm{C} ; R f$ 0.65 (chloroform-ethyl acetate, $8: 1$ ). In the absence of triethylamine, the reduction was completed after $72 \mathrm{hr}$. Anal. Found: C, 67.51; H, 5.86. Calcd. for $\mathrm{C}_{17} \mathrm{H}_{18} \mathrm{O}_{5}: \mathrm{C}$, $67.55 ; \mathrm{H}, 5.96 \%$. NMR $\delta_{\mathrm{C}}\left(\mathrm{CDCl}_{3}\right): 29.20\left(\mathrm{CH}_{2} \beta\right) ; 45.36$ $\left(\mathrm{CH}_{2} \alpha\right) ; 54.88,55.23\left(\right.$ each $\left.\mathrm{OCH}_{3}\right) ; 93.17\left(\mathrm{C}-3^{\prime}, \mathrm{C}-5^{\prime}\right)$; $104.54\left(\mathrm{C}-1^{\prime}\right) ; 113.65(\mathrm{C}-2,6) ; 133.27(\mathrm{C}-1) ; 157.36(\mathrm{C}-4)$; $163.86\left(\mathrm{C}-2^{\prime}, 6^{\prime}\right) ; 165.36\left(\mathrm{C}^{\prime} 4^{\prime}\right) ; 204.48 \quad(\mathrm{C}=\mathrm{O}) . \quad \delta_{\mathrm{H}}$ $\left(\mathrm{CDCl}_{3}\right): 1.60\left(1 \mathrm{H}, \mathrm{s}, 2^{\prime}-\mathrm{OH}\right) ; 2.70 \sim 3.03\left(2 \mathrm{H}, \mathrm{m}, \mathrm{CH}_{2}\right)$; $3.16 \sim 3.40\left(2 \mathrm{H}, \mathrm{m}, \mathrm{CH}_{2}\right) ; 3.70\left(6 \mathrm{H}, \mathrm{s}, 2 \mathrm{OCH}_{3}\right) ; 5.79(2 \mathrm{H}$, $\left.\mathrm{s}, \mathrm{H}-3^{\prime}, 5^{\prime}\right) ; 6.66(2 \mathrm{H}, \mathrm{d}, J=7 \mathrm{~Hz}, \mathrm{H}-3,5) ; 7.00(2 \mathrm{H}, \mathrm{d}, J=$ $7 \mathrm{~Hz}, \mathrm{H}-2,6) ; 9.60\left(1 \mathrm{H}\right.$, br. s, $\left.6^{\prime}-\mathrm{OH}\right)$.

$2^{\prime}$-( $\beta$-D-Glucopyranosyloxy)-6'-hydroxy-4,4'-dimethoxydihydrochalcone (6). To a mixture of $4(285 \mathrm{mg})$ and 5 $(342 \mathrm{mg})$ in dry quinoline $(30 \mathrm{ml})$ was added freshly prepared silver carbonate $(275 \mathrm{mg})$ and $4 \mathrm{~A}$ molecular sieves $(\mathrm{g})$, before the mixture was stirred overnight at room temperature with all light excluded. The reaction mixture was centrifuged and the supernatant was poured into icecooled $10 \%$ acetic acid with stirring. After standing overnight in a refrigerator, the precipitate was collected by filtration, and dissolved in pyridine. The solution was again poured into $10 \%$ acetic acid, and the mixture was treated as just described. The well-dried precipitate was dissolved in $0.1 \mathrm{~N}$ methanolic sodium methoxide $(50 \mathrm{ml})$, and the solution was left overnight at room temperature. After neutralizing with Amberlite IR-I 20 resin $\left(\mathrm{H}^{+}\right)$, the filtered solution was evaporared, and the residual syrup was chromatographed on a silica gel column, using ethyl acetate, saturated with water as the eluent. The fractions containing only 6 were collected and evaporated to a syrup (430 mg), which was crystallized from $50 \%$ ethanol as needles. Yield, $170 \mathrm{mg}(36.0 \%) ; \mathrm{mp} 163^{\circ} \mathrm{C}(50 \%$ ethanol); $[\alpha]_{\mathrm{D}}-46^{\circ}(c=0.5$, ethanol); Rf 0.35 (ethyl acetate sat. with water). Anal. Found: $\mathrm{C}, 59.27 ; \mathrm{H}, 6.00$. Calcd. for $\mathrm{C}_{23} \mathrm{H}_{28} \mathrm{O}_{10}: \mathrm{C}, 59.48 ; \mathrm{H}, 6.03 \%$. Calcd. for $[\mathrm{M}]_{\mathrm{D}}$ (methyl $\alpha$ D-glucoside, +30900$)+[\mathrm{M}]_{\mathrm{D}}(\mathbf{4}, 0):+30900$. Calcd. for $[\mathrm{M}]_{\mathrm{D}}$ (methyl $\beta$-D-glucoside, -6600$)+[\mathrm{M}]_{\mathrm{D}}(4,0):-6600$. Found for $[\mathrm{M}]_{\mathrm{D}}$ : -21334 . The glycosidic linkage of 6 was, therefore, of $\beta$-configuration. Acetate, $\mathrm{mp} 78^{\circ} \mathrm{C}$. Anal. Found: $\mathrm{C}, 58.26 ; \mathrm{H}, 5.61$. Calcd. for $\mathrm{C}_{33} \mathrm{H}_{38} \mathrm{O}_{15}$ : C, 58.75; $\mathrm{H}, 5.64 \%$. NMR $\delta_{\mathrm{H}}\left(\mathrm{CDCl}_{3}\right): 1.93,1.96,2.00,2.05,2.08$ (each s, $3 \mathrm{H}, \mathrm{OAc}) ; 2.70 \sim 3.10\left(1 \mathrm{H}, \mathrm{m}, \mathrm{CH}_{2}-\mathrm{CH}_{2}\right) ; 3.70$ $\left(6 \mathrm{H}, \mathrm{s}, 2 \mathrm{OCH}_{3}\right) ; 4.05 \sim 4.20\left(2 \mathrm{H}\right.$, m, sugar $\left.\mathrm{CH}_{2}\right) ; 4.80 \sim$ $5.10(4 \mathrm{H}$, sugar $\mathrm{H}-2 \sim 5) ; 5.16(1 \mathrm{H}, \mathrm{d}, J=8 \mathrm{~Hz}$, sugar $\mathrm{H}-1)$; $6.30\left(1 \mathrm{H}, \mathrm{d}, J=2 \mathrm{~Hz}, \mathrm{H}-3^{\prime}\right) ; 6.40\left(1 \mathrm{H}, \mathrm{d}, J=2 \mathrm{~Hz}, \mathrm{H}-5^{\prime}\right)$; $6.66(2 \mathrm{H}, \mathrm{d}, J=8 \mathrm{~Hz}, \mathrm{H}-3,5) ; 7.03(2 \mathrm{H}, \mathrm{d}, J=8 \mathrm{~Hz}, \mathrm{H}-$ $2,6)$.

$2^{\prime}-($ Ethoxycarbonyl $)$ methoxy-6'-hydroxy-4, 4'-dimethoxydihydrochalcone (7). To a solution of $4(3.02 \mathrm{~g})$ in DMF $(30 \mathrm{ml})$ were added ethyl monochloracetate $(6 \mathrm{ml})$ and anhydrous potassium carbonate $(1.4 \mathrm{~g})$, before the mixture was stirred for $72 \mathrm{hr}$ at room temperature. The reaction mixture was poured into ice-cooled $1 \%$ hydrochloric acid and extracted with ethyl acetate. The organic layer was washed with water twice and dried $\left(\mathrm{MgSO}_{4}\right)$. After filtration, the filtrate was evaporated and the residue was crystallized from benzene to yield $7(2.23 \mathrm{~g}, 54.5 \%)$ as needles, $\mathrm{mp} 117^{\circ} \mathrm{C}$. Anal. Found: $\mathrm{C}, 64.75 ; \mathrm{H}, 6.21$. Calcd. for $\mathrm{C}_{21} \mathrm{H}_{24} \mathrm{O}_{7}: \mathrm{C}, 64.94 ; \mathrm{H}, 6.19 \%$. NMR $\delta_{\mathrm{C}}\left(\mathrm{CDCl}_{3}\right)$ : $14.10\left(\mathrm{CH}_{3}\right) ; 29.60\left(\mathrm{CH}_{2} \beta\right) ; 45.82\left(\mathrm{CH}_{2} \alpha\right) ; 55.22,55.51$ (each $\left.\mathrm{OCH}_{3}\right) ; 61.53,65.51\left(\right.$ each $\left.\mathrm{CH}_{2}\right) ; 91.41\left(\mathrm{C}-3^{\prime}\right) ; 94.49$ $\left(\mathrm{C}-5^{\prime}\right) ; 105.89\left(\mathrm{C}-1^{\prime}\right) ; 113.76\left(\mathrm{C}^{\prime} 3^{\prime}, 5^{\prime}\right) ; 129.32(\mathrm{C}-2,6)$; $133.70(\mathrm{C}-1) ; 159.79(\mathrm{C}-4) ; 160.43\left(\mathrm{C}^{\prime} 6^{\prime}\right) ; 165.53\left(\mathrm{C}-2^{\prime}\right)$; $167.48(\mathrm{CO}) ; 167.63\left(\mathrm{C}-4^{\prime}\right) ; 204(\mathrm{CO}) . \delta_{\mathrm{H}}\left(\mathrm{CDCl}_{3}\right): 1.23$ $\left(3 \mathrm{H}, \mathrm{t}, J=7 \mathrm{~Hz}, \mathrm{CH}_{3}\right) ; 2.95\left(2 \mathrm{H}, \mathrm{t}, J=6 \mathrm{~Hz}, \mathrm{CH}_{2}\right) ; 3.43$ $\left(2 \mathrm{H}, \mathrm{t}, J=6 \mathrm{~Hz}, \mathrm{CH}_{2}\right) ; 3.73\left(6 \mathrm{H}, \mathrm{s}, 2 \mathrm{OCH}_{3}\right) ; 4.13(2 \mathrm{H}, \mathrm{q}$, $\left.J=7 \mathrm{~Hz}, \mathrm{CH}_{2}\right) ; 4.66\left(2 \mathrm{H}, \mathrm{s},-\mathrm{OCH}_{2} \mathrm{O}-\right) ; 5.70(1 \mathrm{H}, \mathrm{d}, J=$ $\left.2 \mathrm{~Hz}, \mathrm{H}-5^{\prime}\right) ; 6.03\left(1 \mathrm{H}, \mathrm{d}, J=2 \mathrm{~Hz}, \mathrm{H}-3^{\prime}\right) ; 6.73(2 \mathrm{H}, \mathrm{d}, J=$ $8 \mathrm{~Hz}, \mathrm{H}-3,5) ; 7.13(2 \mathrm{H}, \mathrm{d}, J=8 \mathrm{~Hz}, \mathrm{H}-2,6)$.

2'-(Carboxy)methoxy-6'-hydroxy-4,4'-dimethoxydihydrochalcone (8). A suspension of $7(7 \mathrm{~g})$ in $5 \%$ potassium hydroxide $(300 \mathrm{ml})$ was stirred at room temperature until a clear solution was obtained (after about $6 \mathrm{hr}$ ). The solution was acidified with $10 \%$ hydrochloric acid, before the white precipitate was collected by filtration and recrystallized from methanol to afford $8(5.36 \mathrm{~g}, 82.6 \%)$ as needles, mp $173^{\circ} \mathrm{C}$. Anal. Found: C, $43.70 ; \mathrm{H}, 3.82$. Calcd. for $\mathrm{C}_{19} \mathrm{H}_{20} \mathrm{O}_{7}: \mathrm{C}, 43.85 ; \mathrm{H}, 3.85 \%$. NMR $\delta_{\mathrm{C}}\left(\mathrm{DMSO}-d_{6}\right)$ : $29.09\left(\mathrm{CH}_{2} \beta\right) ; 45.11\left(\mathrm{CH}_{2} \propto\right) ; 54.89,55.64\left(\right.$ each $\left.\mathrm{OCH}_{3}\right)$; 
$65.35\left(\mathrm{CH}_{2}\right) ; 91.62\left(\mathrm{C}-3^{\prime}\right) ; 94.29\left(\mathrm{C}-5^{\prime}\right) ; 105.70\left(\mathrm{C}-1^{\prime}\right)$; $113.60(\mathrm{C}-3,5) ; 129.18(\mathrm{C}-2,6) ; 133.18(\mathrm{C}-1) ; 157.36(\mathrm{C}-4)$; $160.57(\mathrm{C}-6) ; 165.34,165.83\left(\mathrm{C}-2^{\prime}, 4^{\prime}\right) ; 169.34(\mathrm{CO}) ; 204.65$ (CO). $\delta_{\mathrm{H}}\left(\mathrm{CDCl}_{3}\right): 2.80\left(2 \mathrm{H}, \mathrm{t}, J=6 \mathrm{~Hz}, \mathrm{CH}_{2}\right) ; 3.33(2 \mathrm{H}, \mathrm{t}$, $\left.J=6 \mathrm{~Hz}, \mathrm{CH}_{2}\right) ; 3.61,3.70\left(\right.$ each $\left.3 \mathrm{H}, \mathrm{s}, \mathrm{OCH}_{3}\right) ; 4.68(2 \mathrm{H}, \mathrm{s}$, $\left.\mathrm{OCH}_{2}\right) ; 5.95\left(2 \mathrm{H}, \mathrm{d}, J=1.5 \mathrm{~Hz}, \mathrm{H}-3^{\prime}, 5^{\prime}\right) ; 6.66(2 \mathrm{H}, \mathrm{d}, J=$ $8 \mathrm{~Hz}, \mathrm{H}-3,5) ; 7.00(2 \mathrm{H}, \mathrm{d}, J=8 \mathrm{~Hz}, \mathrm{H}-2,6)$.

[N-[(6'-Hydroxy-4,4'-dimethoxydihydrochalcone $)-2^{\prime}$ yloxy Jacetylglycyl Jglycine ethyl ester $(\mathbf{1 0})$. To a mixture of $\mathbf{8}(1.44 \mathrm{~g})$ and $9(0.8 \mathrm{~g})$ in DMF $(20 \mathrm{ml})$ were added DEPC $(0.72 \mathrm{ml})$ and triethylamine $(1.12 \mathrm{ml})$, before the solution was stirred for $1 \mathrm{hr}$ at $0^{\circ} \mathrm{C}$ and then allowed to stand overnight at room temperature. The reaction mixture was poured into ice-cooled water with stirring, and the precipitate was collected, washed with water and recrystallized from methanol to give $\mathbf{1 0}(1.5 \mathrm{~g}, 75.0 \%)$ as needles, $\mathrm{mp}$ $165 \sim 167^{\circ} \mathrm{C}$. Anal. Found: C, 59.33; H, 6.02, N, 5.74. Calcd. for $\mathrm{C}_{25} \mathrm{H}_{30} \mathrm{O}_{9} \mathrm{~N}_{2}: \mathrm{C}, 59.76 ; \mathrm{H}, 5.98, \mathrm{~N}, 5.58 \%$. NMR $\delta_{\mathrm{C}}\left(\mathrm{DMSO}-d_{6}\right): 13.99\left(\mathrm{CH}_{3}\right) ; 28.94\left(\mathrm{CH}_{2} \beta\right) ; 45.20$ $\left(\mathrm{CH}_{2} \alpha\right) ; 54.89,55.60\left(\right.$ each $\left.\mathrm{OCH}_{3}\right) ; 60.83\left(\mathrm{CH}_{2}\right) ; 67.46$ $\left(\mathrm{CH}_{2}\right) ; 91.62\left(\mathrm{C}-3^{\prime}\right) ; 94.39\left(\mathrm{C}-5^{\prime}\right) ; 106.01\left(\mathrm{C}-1^{\prime}\right) ; 113.59(\mathrm{C}-$ $3,5) ; 129.17(\mathrm{C}-2,6) ; 133.12(\mathrm{C}-1) ; 157.33(\mathrm{C}-4) ; 160.67$ (C$\left.6^{\prime}\right) ; 165.16\left(\mathrm{C}-2^{\prime}\right) ; 165.31\left(\mathrm{C}-4^{\prime}\right) ; 167.19,168.67,169.54$, 204.52 (each CO). $\delta_{\mathrm{H}}$ (DMSO- $\left.d_{6}\right): 1.16(3 \mathrm{H}, \mathrm{t}, J=6 \mathrm{~Hz}$, $\left.\mathrm{CH}_{3}\right) ; 2.63 \sim 2.93\left(4 \mathrm{H}, \mathrm{m},-\mathrm{CH}_{2}-\mathrm{CH}_{2}\right) ; 3.36,3.70$ (each $\left.3 \mathrm{H}, \mathrm{s}, \mathrm{OCH}_{3}\right) ; 4.00\left(2 \mathrm{H}, J=6 \mathrm{~Hz}, \mathrm{CH}_{2}\right) ; 4.60(2 \mathrm{H}, \mathrm{s}$, $\left.\mathrm{OCH}_{2} \mathrm{CO}\right) ; 5.95\left(2 \mathrm{H}, \mathrm{d}, J=1.5 \mathrm{~Hz}, \mathrm{H}-3^{\prime}, 5^{\prime}\right) ; 6.66(2 \mathrm{H}, \mathrm{d}$, $J=8 \mathrm{~Hz}, \mathrm{H}-3,5) ; 7.00(2 \mathrm{H}, \mathrm{d}, J=8 \mathrm{~Hz}, \mathrm{H}-2,6) ; 8.00 \sim 8.27$ $(2 \mathrm{H}, \mathrm{m}, 2 \mathrm{NH}) ; 13.2(1 \mathrm{H}, \mathrm{s}, \mathrm{OH})$.

IN-[(6'-Hydroxy-4,4'-dimethoxydihydrochalcone $)-2^{\prime}$ yloxy Jacetylglycyl Jglycine (11). To a solution of $10(1.4 \mathrm{~g})$ in DMF ( $32 \mathrm{ml}$ ) was added $1 \mathrm{~N}$ sodium hydroxide solution ( $7 \mathrm{ml}$ ), before the mixture was stirred for $1.5 \mathrm{hr}$ at room temperature. After the reaction was complete, the mixture was acidified with $4 \%$ hydrochloric acid. The precipitate was collected and recrystallized from methanol or ethyl acetate to give 11 (almost quantitative) as needles, $\mathrm{mp}$ $180 \sim 181^{\circ} \mathrm{C}$. Anal. Found: C, 58.06; H, 5.46; N, 5.78 . Caled. for $\mathrm{C}_{23} \mathrm{H}_{26} \mathrm{~N}_{2} \mathrm{O}_{9}: \mathrm{C}, 58.23 ; \mathrm{H}, 5.49 ; \mathrm{N}, 5.91 \% . R f$ 0.56 (chloroform-methanol-water, 7:5:1). NMR $\delta_{\mathrm{C}}$ $\left(\mathrm{DMSO}-d_{6}\right): 29.05\left(\mathrm{CH}_{2} \beta\right) ; 40.71,41.65\left(\right.$ each $\left.\mathrm{CH}_{2}\right) ; 45.33$ $\left(\mathrm{CH}_{2} \alpha\right) ; 54.97,55.67\left(\right.$ each $\left.\mathrm{OCH}_{3}\right) ; 67.56(\mathrm{C}-3) ; 91.71(\mathrm{C}-$ $\left.3^{\prime}\right) ; 94.50\left(\mathrm{C}-5^{\prime}\right) ; 106.04\left(\mathrm{C}-1^{\prime}\right) ; 113.70(\mathrm{C}-3,5) ; 129.27$ (C$2,6) ; 133.24$ (C-9); 157.44 (C-4); 160.81 (C-6'); 165.29 (C$2) ; 165.56\left(\mathrm{C}-4^{\prime}\right) ; 167.31,168.82,171.06,204.62$ (each CO). $\delta_{\mathrm{H}}\left(\mathrm{DMSO}-d_{6}\right): 2.90\left(2 \mathrm{H}, \mathrm{t}, J=6 \mathrm{~Hz}, \mathrm{CH}_{2}\right) ; 3.40(2 \mathrm{H}$, t. $\left.J=6 \mathrm{~Hz}, \mathrm{CH}_{2}\right) ; 3.70,3.80\left(\right.$ each $\left.3 \mathrm{H}, \mathrm{OCH}_{3}\right) ; 4.70(2 \mathrm{H}, \mathrm{s}$, $\left.-\mathrm{OCH}_{2} \mathrm{CO}\right) ; 3.50 \sim 4.00\left(4 \mathrm{H}, \mathrm{m}, 2 \mathrm{NH}-\mathrm{CH}_{2}-\mathrm{CO}\right) ; 6.03$ $\left(2 \mathrm{H}, \mathrm{d}, J=2 \mathrm{~Hz}, \mathrm{H}-3^{\prime}, 5^{\prime}\right) ; 6.76(2 \mathrm{H}, \mathrm{d}, J=8 \mathrm{~Hz}, \mathrm{H}-3,5)$; $7.13(2 \mathrm{H}, \mathrm{d}, J=8 \mathrm{~Hz}, \mathrm{H}-2,6) ; 8.23(2 \mathrm{H}, \mathrm{t}, J=4 \mathrm{~Hz}, 2 \mathrm{NH})$.

$N^{\alpha}$-Benzyloxycarbonyl- $N^{\varepsilon}-\left[\left(6^{\prime}-h y d r o x y-4,4^{\prime}\right.\right.$-dimethoxydihydrochalcone)-2'-yloxy ] acet yl-L-lysine benzyl ester (13). To a mixture of $8(1.31 \mathrm{~g})$ and $12(1.93 \mathrm{~g})$ in DMF $(25 \mathrm{ml})$, DEPC $(0.62 \mathrm{ml})$ and triethylamine $(1 \mathrm{ml})$ were added, before the mixture was allowed to stand for $1 \mathrm{hr}$ at $0^{\circ} \mathrm{C}$ and then overnight at room temperature. After the reaction was complete, the solution was poured into ice-cooled water with stirring, and the precipitate was extracted with ethyl acetate. The organic layer was washed successively with $4 \%$ hydrochloric acid, $4 \%$ sodium hydrogencarbonate solution and water, and then dried $\left(\mathrm{MgSO}_{4}\right)$ and evaporated to a residue, which was recrystalized from ethanol to give $13(1.25 \mathrm{~g}, 48.3 \%)$ as needles, mp $139 \sim$ $141{ }^{\circ} \mathrm{C}$. Rf 0.50 (chloroform-ethyl acetate, 2:1). Anal. Found: C, 67.42; H, 6.18; N, 3.98. Calcd. for $\mathrm{C}_{40} \mathrm{H}_{44} \mathrm{~N}_{2} \mathrm{O}_{10}: \mathrm{C}, 67.42 ; \mathrm{H}, 6.18 ; \mathrm{N}, 3.93 \%$. NMR $\delta_{\mathrm{C}}$ $\left(\mathrm{DMSO}-d_{6}\right)$ : $22.81,28.45\left(\mathrm{each} \mathrm{CH}_{2}\right) ; 28.99\left(\mathrm{CH}_{2} \beta\right)$; $30.30,38.33\left(\right.$ each $\left.\mathrm{CH}_{2}\right) ; 45.27\left(\mathrm{CH}_{2} \alpha\right) ; 53.98(\mathrm{CH}), 54.88$, 55.58 (each $\left.\mathrm{OCH}_{3}\right) ; 65.48,65.84$ (each $\left.\mathrm{PhCH}_{2}\right) ; 67.67$ $(\mathrm{CH}), 91.63\left(\mathrm{C}-3^{\prime}\right) ; 94.26\left({\left.\mathrm{C}-5^{\prime}\right)}_{1} ; 06.00\left(\mathrm{C}-1^{\prime}\right) ; 113.57(\mathrm{C}-\right.$ $3,5) ; 127.40,127.66,127.92,128.24$ (each $\mathrm{PhCH}_{2}-2 \sim 6$ ); $128.30 \quad(\mathrm{C}-2,6), \quad 133.07 \quad(\mathrm{C}-1), \quad 135.85,136.78 \quad$ (each

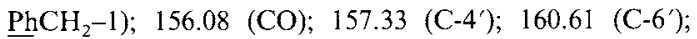
$165.11,165.34\left({\mathrm{C}-2^{\prime}}^{\prime} 4^{\prime}\right) ; 166.55,172.18,204.51$ (each CO). $\delta_{\mathrm{H}}\left(\mathrm{CDCl}_{3}\right) .0 .80 \sim 1.85\left(6 \mathrm{H}, \mathrm{m}, 3 \mathrm{CH}_{2}\right): 2.66 \sim 3.50(\mathrm{~m}$, $\left.6 \mathrm{H}, 3 \mathrm{CH}_{2}\right) ; 3.70,3.73\left(\right.$ each $\left.3 \mathrm{H}, \mathrm{s}, \mathrm{OCH}_{3}\right) ; 5.03,5.10($ each $\left.2 \mathrm{H}, \mathrm{s}, \mathrm{PhCH}_{2}\right) ; 6.78$ (1H, NH); 6.08 ( $2 \mathrm{H}$, br.s, H-3', 5'); $6.75(2 \mathrm{H}, \overline{\mathrm{d}}, J=8 \mathrm{~Hz}, \mathrm{H}-3,5) ; 7.10(2 \mathrm{H}, \mathrm{d}, J=8 \mathrm{~Hz}, \mathrm{H}-$ $2,6) ; 7.33\left(10 \mathrm{H}, \mathrm{s}, 2 \underline{\mathrm{PhCH}}_{2}\right)$.

$N^{\varepsilon}-\int\left(6^{\prime}-H y d r o x y-4,4^{\prime}\right.$-dimethoxydihydrochalcone $)-2^{\prime}-$ yloxy Jacetyl-L-lysine (14). A mixture of $13(500 \mathrm{mg})$ and $10 \% \mathrm{Pd}-\mathrm{C}(500 \mathrm{mg})$ in acetic acid $(15 \mathrm{ml})$ was stirred under a hydrogen atmosphere for $7.5 \mathrm{hr}$ at room temperature. The catalyst was filtered off through a celite bed, and the filtrate was evaporated to give 14 as long needles ( $263 \mathrm{mg}$, $70.3 \%)$, mp $173 \sim 175^{\circ} \mathrm{C},[\alpha]_{\mathrm{D}}+10^{\circ}(c=1$, acetic acid $) . R f$ 0.82 (ethanol-acetic acid-water, $3: 2: 1$ ). The ninhydrin test was positive. Anal. Found: C, $56.29 ; \mathrm{H}, 6.17 ; \mathrm{N}, 5.13$. Calcd for $\mathrm{C}_{25} \mathrm{H}_{32} \mathrm{~N}_{2} \mathrm{O}_{8} .2 \frac{1}{2} \mathrm{H}_{2} \mathrm{O}: \mathrm{C}, 56.28 ; \mathrm{H}, 6.94 ; \mathrm{N}$, 5.25\%. NMR $\delta_{\mathrm{C}}\left(\mathrm{CD}_{3} \mathrm{COOD}\right): 16.00,19.95,20.66,20.10$ (each $\left.\mathrm{CH}_{2}, \mathrm{CH}_{2} \beta\right) ; 30.00\left(\mathrm{CH}_{2}\right) ; 36.65\left(\mathrm{CH}_{2} \alpha\right) ; 46.00$, $46.30\left(\right.$ each $\left.\mathrm{OCH}_{3}\right) ; 58.50\left(\mathrm{CH}_{2} \alpha, \mathrm{CH}\right) ; 83.50\left(\mathrm{C}-3^{\prime}\right)$; $86.00\left(\mathrm{C}-5^{\prime}\right) ; 105.00\left(\mathrm{C}-3,5,1^{\prime}\right) ; 124.60(\mathrm{C}-1) ; 148.50(\mathrm{C}-4)$; $151.70\left(\mathrm{C}-6^{\prime}\right) ; 157.00\left(\mathrm{C}-4^{\prime}, 2^{\prime}\right) ; 158.00,168.50,197.50$ (each $\mathrm{CO}) . \delta_{\mathrm{H}}\left(\mathrm{CD}_{3} \mathrm{COOD}\right): 1.05 \sim 2.10\left(\mathrm{~m}\right.$, lysine $\left.\mathrm{CH}_{2}\right)$; $2.70 \sim 3.39\left(\mathrm{~m}, \mathrm{CH}_{2}-\mathrm{CH}_{2}\right.$, lysine $\left.\mathrm{CH}_{2}\right) ; 3.60,3.64$ (each $\left.3 \mathrm{H}, \mathrm{s}, \mathrm{OCH}_{3}\right) ; 4.50\left(2 \mathrm{H}, \mathrm{s}, \mathrm{OCH}_{2} \mathrm{CO}\right) ; 5.76\left(1 \mathrm{H}, \mathrm{s}, \mathrm{H}-3^{\prime}\right)$; $5.94(1 \mathrm{H}, \mathrm{s}, \mathrm{H}-5) ; 6.57(2 \mathrm{H}, \mathrm{d}, J=8 \mathrm{~Hz}, \mathrm{H}-3,5) ; 6.90(2 \mathrm{H}$, d, $J=6 \mathrm{~Hz}, \mathrm{H}-2,6)$.

$N$-Benzyloxycarbonyl-O- $\left[\left(6^{\prime}-\right.\right.$ hydroxy- $4,4^{\prime}$-dimethoxydihydrochalcone)-2'-yljhomoserine methyl ester (16). A mixture of $4(4.05 \mathrm{~g}), 15(4.41 \mathrm{~g})$ and anhydrous potassium carbonate $(2.04 \mathrm{~g})$ in DMF $(70 \mathrm{ml})$ was stirred for $72 \mathrm{hr}$ at room temperature. The reaction mixture was poured into ice-cooled water $(600 \mathrm{ml})$ with stirring, acidified with $10 \%$ hydrochloric acid, and then extracted with ethyl acetate. The organic layer was washed with water, dried $\left(\mathrm{MgSO}_{4}\right)$ and evaporated. The residue was crystallized from methanol to give 16 as needles $(4.5 \mathrm{~g}, 60.9 \%)$ mp $115 \sim 116^{\circ} \mathrm{C}$ 
(methanol). Rf 0.52 (chloroform-ethyl acetate, 10:1). Anal. Found: C, 65.30; H, 6.01; N, 2.52. Calcd. for $\mathrm{C}_{30} \mathrm{H}_{33} \mathrm{NO}_{9}: \mathrm{C}, 65.33 ; \mathrm{H}, 5.99 ; \mathrm{N}, 2.54 \%$. NMR $\delta_{\mathrm{C}}$ $\left(\mathrm{CDCl}_{3}\right): 28.77\left(\mathrm{CH}_{2} \beta\right) ; 30.09\left(\mathrm{CH}_{2}\right) ; 45.15\left(\mathrm{CH}_{2} \alpha\right)$; $50.91\left(\mathrm{CH}_{2}\right) ; 51.89\left(\mathrm{CH}_{3}\right) ; 54.82,55.53\left(\right.$ each $\left.\mathrm{OCH}_{3}\right)$;

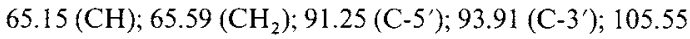
$\left(\mathrm{C}-1^{\prime}\right) ; 113.54(\mathrm{C}-3,5) ; 127.54,127.69,128.18,129.02$ $\left(\mathrm{PhCH}_{2}-2 \sim 6\right) ; 132.95(\mathrm{C}-1) ; 136.72\left(\mathrm{PhCH}_{2}-1\right) ; 156.05$ (CO); $157.29(\mathrm{C}-4) ; 161.32\left(\mathrm{C}^{\prime} 6^{\prime}\right) ; 165.43,165.80\left(\mathrm{C}-2^{\prime}, 4^{\prime}\right)$; $172.12,204.10($ each $\mathrm{CO}) . \delta_{\mathrm{H}}\left(\mathrm{CDCl}_{3}\right): 1.85 \sim 2.60(2 \mathrm{H}, \mathrm{m}$, $\left.-\mathrm{OCH}_{2} \mathrm{CH}_{2}-\right) ; 2.70 \sim 3.43\left(4 \mathrm{H}, \mathrm{m}, \mathrm{Ar}-\mathrm{CH}_{2}-\mathrm{CH}_{2} \mathrm{CO}\right) ; 3.60$ $\left(3 \mathrm{H}, \mathrm{s}, \mathrm{COCH}_{3}\right) ; 3.70,3.75\left(\right.$ each $\left.3 \mathrm{H}, \mathrm{s}, \mathrm{OCH}_{3}\right) ; 4.00(2 \mathrm{H}$, $\left.\mathrm{t}, J=6 \mathrm{~Hz}, \mathrm{OCH}_{2} \mathrm{CH}_{2}\right) ; 4.43(1 \mathrm{H}, \mathrm{d}, J=6 \mathrm{~Hz}, \mathrm{C}-\mathrm{CH}-\mathrm{N})$; $5.03\left(2 \mathrm{H}, \mathrm{s}, \mathrm{PhCH}_{2}\right) ; 5.33(1 \mathrm{H}, \mathrm{d}, J=6 \mathrm{~Hz},-\mathrm{NH}) ; 5.80$ $\left(1 \mathrm{H}, \mathrm{d}, J=2 \mathrm{~Hz}, \overline{\mathrm{H}}-3^{\prime}\right) ; 6.00\left(1 \mathrm{H}, \mathrm{d}, J=2 \mathrm{~Hz}, \mathrm{H}-5^{\prime}\right) ; 6.70$ $(2 \mathrm{H}, \mathrm{d}, J=8 \mathrm{~Hz}, \mathrm{H}-3,5) ; 7.06(2 \mathrm{H}, \mathrm{d}, J=8 \mathrm{~Hz}, \mathrm{H}-2,6)$; $7.25\left(5 \mathrm{H}, \mathrm{s}, \underline{\mathrm{PhCH}_{2}}\right)$.

$N$-Benzyloxycarbonyl-O- $\left[\left(6^{\prime}\right.\right.$-hydroxy-4, $4^{\prime}$-dime thoxydihydrochalcone)-2'-yl]homoserine (17). To a suspension of $16(4 \mathrm{~g})$ in $4 \%$ sodium hydroxide solution $(100 \mathrm{ml})$ was added acetone (about $100 \mathrm{ml}$ ) until a clear solution was obtained. After standing for $2 \mathrm{hr}$ at room temperature, the reaction mixture was neutralized with $4 \%$ hydrochloric acid with ice-cooling. To this, actone $(80 \mathrm{ml})$ was added until the syrup deposited was completely dissolved, and then the solution was acidified with $4 \%$ hydrochloric acid. After standing for a while, the crystals deposited were collected by filtration and recrystallized from ethanol to yield $3.6 \mathrm{~g}(92.3 \%)$ of 17 as needles, mp $142 \sim 143^{\circ} \mathrm{C}$. Anal. Found: $\mathrm{C}, 64.68 ; \mathrm{H}, 5.77 ; \mathrm{N}, 2.63$. Calcd. for $\mathrm{C}_{29} \mathrm{H}_{31} \mathrm{NO}_{9}$ : $\mathrm{C}, 64.80 ; \mathrm{H}, 5.77 ; \mathrm{N}, 2.61 \%$. NMR $\delta_{\mathrm{C}}\left(\mathrm{DMSO}-d_{6}\right): 28.67$ $\left(\mathrm{CH}_{2} \beta\right) ; 30.16\left(\mathrm{CH}_{2}\right) ; 44.86\left(\mathrm{CH}_{2} \alpha\right) ; 50.86\left(\mathrm{CH}_{2}\right) ; 54.82$, $55.53\left(\right.$ each $\left.\mathrm{OCH}_{3}\right) ; 65.41\left(\mathrm{CH}_{2}, \mathrm{CH}\right), 91.23\left(\mathrm{C}-5^{\prime}\right) ; 93.85$ $\left(\mathrm{C}-3^{\prime}\right) ; 105.64\left(\mathrm{C}-1^{\prime}\right) ; 113.55(\mathrm{C}-3,5) ; 127.49,127.60$, $128.13,129.98\left(\mathrm{C}-2,6 ; \mathrm{PhCH}_{2}-2 \sim 6\right) ; 132.89(\mathrm{C}-1) ; 136.78$ $\left.\left(\mathrm{PhCH}_{2}-1\right) ; 156.04(\mathrm{CO}) ; 157.24(\mathrm{C}-4) ; 161.28\left(\mathrm{C}^{\prime}\right)^{\prime}\right)$;

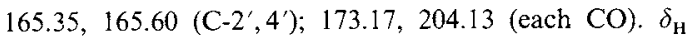
$\left(\mathrm{DMSO}-d_{6}\right): 1.66 \sim 2.60\left(2 \mathrm{H}, \mathrm{m}, \mathrm{OCH}_{2} \mathrm{CH}_{2}\right) ; 2.63 \sim 3.50$ $\left(4 \mathrm{H}, \mathrm{m}, \mathrm{PhCH}_{2} \mathrm{CH}_{2} \mathrm{CO}\right.$ ); 3.63, 3.73 (each $3 \mathrm{H}, \mathrm{s}, \mathrm{OCH}_{3}$ ); $3.86 \sim 4.32\left(3 \mathrm{H}, \mathrm{m}, \mathrm{OCH}_{2} \mathrm{CH}_{2}, \mathrm{CHNH}\right) ; 4.96(2 \mathrm{H}, \mathrm{s}$, $\left.\mathrm{PhCH}_{2}\right) ; 6.00\left(2 \mathrm{H}, \mathrm{s}, \mathrm{H}-3^{\prime}, 5^{\prime}\right) ; 6.70(2 \mathrm{H}, \mathrm{d}, J=8 \mathrm{~Hz}, \mathrm{H}-$

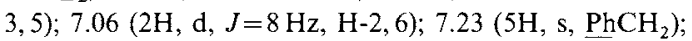
$7.63(1 \mathrm{H}, \mathrm{d}, J=8 \mathrm{~Hz}, \mathrm{NH})$

$O-\left[\left(6^{\prime}-H y d r o x y-4,4^{\prime}\right.\right.$-dimethoxydihydrochalcone $)-2$ '-yl]homoserine (18). A mixture of $17(1 \mathrm{~g})$ and $10 \% \mathrm{Pd}-\mathrm{C}$ $(500 \mathrm{mg})$ in tetrahydrofuran $(20 \mathrm{ml})$ containing acetic acid $(2 \mathrm{ml})$ was stirred under hydrogen for $6 \mathrm{hr}$, and then treated as described for the preparation of 14 from 13 to afford 18 as needles $(680 \mathrm{mg}, 90.7 \%), \mathrm{mp} 189 \sim 191^{\circ} \mathrm{C}$. Anal. Found: C, 62.77; H, 5.98; N, 3.65. Calcd. for $\mathrm{C}_{21} \mathrm{H}_{25} \mathrm{NO}_{7}$ : C, 62.53; H, 6.20; N, 3.47\%.

Compound $18(419 \mathrm{mg})$ was dissolved by heating in $70 \mathrm{ml}$ of $1 \mathrm{~N}$ hydrochloric acid. After being cooled to ambient temperature, the crystallization solution was filtered, and the crystals were allowed to air dry to yield
$370 \mathrm{mg}(80.5 \%)$ of the hydrochloride salt $\left(\mathbf{1 8}^{\prime}\right)$ as long needles, $m p 185^{\circ} \mathrm{C}$ (sint. $180^{\circ} \mathrm{C}$ )

[N-Benzyloxycarbonyl-O-[(6'-hydroxy-4,4'-dimethoxydihydrochalcone)-2'-yl Jhomoseryl Jglycine benzyl ester (20). To a mixture of $17(2.42 \mathrm{~g})$ and $19(1.52 \mathrm{~g})$ in DMF $(25 \mathrm{ml})$ were added DEPC $(0.72 \mathrm{ml})$ and triethylamine $(1.26 \mathrm{ml})$, before the mixture was stood for $1 \mathrm{hr}$ at $0^{\circ} \mathrm{C}$ and then overnight at room temperature. Ice-cooled water was added to the reaction mixture, and the white viscous oil deposited was extracted with a large quantity of ethyl acetate. The organic layer was treated as described for the preparation of $\mathbf{1 3}$ from $\mathbf{8}$, and the final crystalline residue was recrystallized from ethanol to give $\mathbf{2 0}$ as needles $(2.56 \mathrm{~g}, 82.6 \%), \mathrm{mp} 150 \sim 153^{\circ} \mathrm{C}$. Anal. Found: C, 64.50; $\mathrm{H}, 5.30 ; \mathrm{N}, 5.20$. Calcd. for $\mathrm{C}_{29} \mathrm{H}_{30} \mathrm{~N}_{2} \mathrm{O}_{9}: \mathrm{C}, 64.27 ; \mathrm{H}$, $5.45 ; \mathrm{N}, 5.09 \%$. NMR $\delta_{\mathrm{C}}\left(\mathrm{DMSO}-d_{6}\right): 28.77\left(\mathrm{CH}_{2} \beta\right)$; $31.03,41.35\left(\right.$ each $\left.\mathrm{CH}_{2}\right) ; 44.90\left(\mathrm{CH}_{2} \alpha\right) ; 51.80\left(\mathrm{CH}_{2}\right)$; $54.83,55.56\left(\right.$ each $\left.\mathrm{OCH}_{3}\right) ; 65.24(\mathrm{CH}) ; 65.48,65.79$ (each $\left.\left.\mathrm{CH}_{2}\right) ; 91.24\left(\mathrm{C}-3^{\prime}\right) ; 93.80\left(\mathrm{C}-5^{\prime}\right) ; 105.72\left(\mathrm{C}^{\prime}\right)^{\prime}\right) ; 113.56(\mathrm{C}-$ $3,5) ; 126.70 \sim 128.26 \quad\left(2 \mathrm{PhCH}_{2}-2 \sim 6\right) ; 129.05 \quad(\mathrm{C}-2,6)$; $132.98(\mathrm{C}-1) ; 135.78,136.75\left(2 \mathrm{PhCH}_{2}-1\right) ; 155.81(\mathrm{CO})$; 157.24 (C-4); 161.35 (C-6'); 165.37 (C-2'); 165.54 (C-4'); $169.45,171.73,204.31$ (each CO). $\delta_{\mathrm{H}}\left(\mathrm{CDCl}_{3}\right): 1.80 \sim 4.33$ $\left(13 \mathrm{H}, \mathrm{m},-\mathrm{OCH}_{2} \mathrm{CH}_{2} \mathrm{CH}(\mathrm{NH}) \mathrm{CONHCH}_{2}, \mathrm{PhCH}_{2} \mathrm{CH}_{2}-\right.$ $\mathrm{CO}) ; 3.63,3.80\left(\right.$ each $\left.3 \mathrm{H}, \mathrm{s}, \mathrm{OCH}_{3}\right) ; 5.00,5.02($ each $2 \mathrm{H}, \mathrm{s}$, $\left.\mathrm{PhCH}_{2}\right) ; 6.03\left(2 \mathrm{H}, \mathrm{s}, \mathrm{H}-3^{\prime}, 5^{\prime}\right) ; 6.73(2 \mathrm{H}, \mathrm{d}, J=8 \mathrm{~Hz}, \mathrm{H}-$ $3,5) ; 7.06(2 \mathrm{H}, \mathrm{d}, J=8 \mathrm{~Hz}, \mathrm{H}-2,6) ; 7.30\left(10 \mathrm{H}, \mathrm{s}, 2 \mathrm{PhCH}_{2}\right)$

[O-[(6'-Hydroxy-4,4'-dimethoxydihydrochalcone)- $2^{\prime}$ $y l /$ homoseryl /glycine (21). A mixture of benzyl ester (20, $1 \mathrm{~g})$ and $10 \% \mathrm{Pd}-\mathrm{C}(600 \mathrm{mg})$ in warm acetic acid $(35 \mathrm{ml}$, $50^{\circ} \mathrm{C}$ ) was stirred under hydrogen for $2 \mathrm{hr}$ at $50^{\circ} \mathrm{C}$ and then for $3 \mathrm{hr}$ at room temperature. The reaction mixture was treated in the same way as described for the preparation of 14 from 13 to give a syrup, which was crystallized from ethanol to give $21(660 \mathrm{mg}, 98.5 \%$ ) as needles, $\mathrm{mp}$ $114 \sim 118^{\circ} \mathrm{C}$. Anal. Found: C, 59.50; H, 6.38; N, 5.86 . Calcd. for $\mathrm{C}_{23} \mathrm{H}_{28} \mathrm{~N}_{2} \mathrm{O}_{8}: \mathrm{C}, 60.00 ; \mathrm{H}, 6.09 ; \mathrm{N}, 6.09 \%$. NMR $\delta_{\mathrm{C}}\left(\mathrm{CD}_{3} \mathrm{CO}_{2} \mathrm{D}\right): 30.44\left(\mathrm{CH}_{2} \beta\right) ; 31.60,42.30$ (each $\left.\mathrm{CH}_{2}\right) ; 46.50\left(\mathrm{CH}_{2} \alpha\right) ; 52.30\left(\mathrm{CH}_{2}\right) ; 55.57,56.10$ (each $\left.\mathrm{OCH}_{3}\right) ; 65.44(\mathrm{CH}) ; 92.95\left(\mathrm{C}-3^{\prime}\right) ; 95.13\left(\mathrm{C}-5^{\prime}\right) ; 106.89(\mathrm{C}-$ $\left.1^{\prime}\right) ; 114.78(\mathrm{C}-3,5) ; 130.22(\mathrm{C}-2,6) ; 134.60(\mathrm{C}-1) ; 158.84$

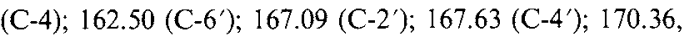
177.73, 205.91 (each CO). $\delta_{\mathrm{H}}\left(\mathrm{CD}_{3} \mathrm{CO}_{2} \mathrm{D}\right): 2.64 \sim 3.44$ $\left(4 \mathrm{H}, \mathrm{m}, \mathrm{PhCH}_{2} \mathrm{CH}_{2} \mathrm{CO}\right) ; 2.12 \sim 4.46\left(\mathrm{~m},-\mathrm{OCH}_{2} \mathrm{CH}_{2} \mathrm{CH}-\right.$ $\left.\mathrm{CONHCH}_{2}\right) ; 3.66,3.70$ (each $\left.3 \mathrm{H}, \mathrm{OCH}_{3}\right) ; 6 . \overline{00}(\overline{2 \mathrm{H}}, \overline{\mathrm{m}}$, $\left.\mathrm{H}-3^{\prime}, 5^{\prime}\right) ; \overline{6} .66(2 \mathrm{H}, \mathrm{d}, J=8 \mathrm{~Hz}, \mathrm{H}-3,5) ; 7.00(2 \mathrm{H}, \mathrm{d}, J=8 \mathrm{~Hz}$, $\mathrm{H}-2,6)$.

$N$-Benzyloxycarbonyl-/O-/(6'-hydroxy-4, $4^{\prime}$-dimethoxydihydrochalcone)-2'-yl homoseryl /glycine ethyl ester (22). To a mixture of $17(1.61 \mathrm{~g})$ and glycine ethyl ester hydrochloride $(0.41 \mathrm{~g})$ in DMF $(15 \mathrm{ml})$ were added DEPC $(0.48 \mathrm{~g})$ and triethylamine $(0.84 \mathrm{ml})$, before the mixture was treated in the same way as that described for the preparation of $\mathbf{2 0}$. The final syrup was crystallized from 
methanol to give 22 as plates $\left(1.82 \mathrm{~g}, 97.9^{\circ}\right), \mathrm{mp} 143^{\circ} \mathrm{C}$ (methanol). Rf 0.16 (chloroform-ethyl acetate, 10:1). Anal. Found: C, 63.58; H, 6.03; N, 4.48. Calcd. for $\mathrm{C}_{33} \mathrm{H}_{38} \mathrm{~N}_{2} \mathrm{O}_{10}: \mathrm{C}, 63.66 ; \mathrm{H}, 6.11 ; \mathrm{N}, 4.50 \%$. NMR $\delta_{\mathrm{C}}$ $\left(\mathrm{CDCl}_{3}\right): 13.97\left(\mathrm{CH}_{3}\right) ; 28.84\left(\mathrm{CH}_{2} \beta\right) ; 31.14,40.81$ (each $\left.\mathrm{CH}_{2}\right) ; 44.98\left(\mathrm{CH}_{2} \alpha\right) ; 51.80\left(\mathrm{CH}_{2}\right) ; 54.83,55.50$ (each $\left.\mathrm{OCH}_{3}\right) ; 60.42\left(\mathrm{CH}_{2}\right) ; 65.57\left(\mathrm{CH}, \mathrm{CH}_{2}\right) ; 91.27\left(\mathrm{C}-3^{\prime}\right) ; 93.86$ $\left(\mathrm{C}-5^{\prime}\right) ; 105.72\left(\mathrm{C}-1^{\prime}\right) ; 113.59(\mathrm{C}-3,5) ; 127.57,127.67$, 127.85, $128.21\left(\mathrm{PhCH}_{2}-2 \sim 6\right) ; 129.09(\mathrm{C}-2,6) ; 133.04$ (C1); $136.81\left(\mathrm{PhCH}_{2}-1\right) ; 155.87(\mathrm{C}=\mathrm{O}) ; 157.32(\mathrm{C}-4) ; 161.46$ $\left(\mathrm{C}^{\prime} 6^{\prime}\right) ; 165 . \overline{47}\left(\mathrm{C}-2^{\prime}\right) ; 165.75\left(\mathrm{C}^{\prime} 4^{\prime}\right) ; 169.54,171.74,204.36$ (each $\mathrm{CO}) . \delta_{\mathrm{H}}\left(\mathrm{CDCl}_{3}\right): 1.16\left(3 \mathrm{H}, \mathrm{t}, J=6 \mathrm{~Hz}, \mathrm{CH}_{3}\right) ; 4.06$ $\left(2 \mathrm{H}, \mathrm{q}, J=6 \mathrm{~Hz}, \mathrm{CH}_{2}\right) ; 1.70 \sim 4.50\left(\mathrm{~m}, \mathrm{OCH}_{2} \mathrm{CH}_{2} \mathrm{CH}-\right.$ $\left.\mathrm{CONHCH}_{2}\right) ; 3.75,3.80\left(6 \mathrm{H}\right.$, each $\left.3 \mathrm{H}, \mathrm{s}, 2 \overline{\mathrm{OCH}_{3}}\right) ;$ $5.00\left(2 \mathrm{H}, \mathrm{s}, \mathrm{PhCH}_{2}\right) ; 6.03\left(2 \mathrm{H}, \mathrm{s}, \mathrm{H}-3^{\prime}, 5^{\prime}\right) ; 6.73(2 \mathrm{H}, \mathrm{d}, J=$ $8 \mathrm{~Hz}, \mathrm{H}-3,5) ; 7.10(2 \mathrm{H}, \mathrm{d}, J=8 \mathrm{~Hz}, \mathrm{H}-2,6) ; 7.26(5 \mathrm{H}, \mathrm{s}$, $\left.\mathrm{PhCH}_{2}\right) ; 7.60(1 \mathrm{H}, \mathrm{d}, J=6 \mathrm{~Hz}, \mathrm{NH}) ; 8.33(1 \mathrm{H}, \mathrm{t}, J=6 \mathrm{~Hz}$, $\mathrm{NH}) ; 13.52\left(1 \mathrm{H}, \mathrm{s}, 6^{\prime}-\mathrm{OH}\right)$.

IN-Benzyloxycarbonyl-[O-(6'-hydroxy-4,4'-dimethoxydihydrochalcone)-2'-yl Jhomoseryl Jglycine (23). To a solution of $22(1 \mathrm{~g})$ in DMF $(10 \mathrm{ml})$ was added $1 \mathrm{~N}$ sodium hydroxide solution $(2 \mathrm{ml})$, and the solution was allowed to stand overnight at room temperature. The solution was poured into ice-cooled $1 \%$ hydrochloric acid and extracted with ethyl acetate. The extract was washed with water, dried over $\mathrm{MgSO}_{4}$ and evaporated. The residual syrup was crystallized from ethanol-ether to give $23(515 \mathrm{mg}, 53.9 \%)$, mp $157 \sim 159^{\circ} \mathrm{C}$. Anal. Found: C, 61.92; H, 5.76; N, 4.67. Calcd. for $\mathrm{C}_{31} \mathrm{H}_{34} \mathrm{~N}_{2} \mathrm{O}_{12}: \mathrm{C}, 62.63 ; \mathrm{H}, 5.72 ; \mathrm{N}, 4.71 \%$. NMR $\delta_{\mathrm{C}}\left(\mathrm{DMSO}-d_{6}\right): 28.79\left(\mathrm{CH}_{2} \beta\right) ; 31.12,41.36$ (each $\left.\mathrm{CH}_{2}\right) ; 44.93\left(\mathrm{CH}_{2} \alpha\right) ; 51.75\left(\mathrm{CH}_{2}\right) ; 54.85,55.58$ (each $\left.\mathrm{OCH}_{3}\right) ; 65.50\left(\mathrm{CH}_{2}, \mathrm{CH}\right) ; 91.28\left(\mathrm{C}-3^{\prime}\right) ; 93.82\left(\mathrm{C}-5^{\prime}\right)$; $105.72\left(\mathrm{C}-1^{\prime}\right) ; 113.57(\mathrm{C}-3,5) ; 127.52,127.64\left(\mathrm{PhCH}_{2}-\right.$ $2 \sim 6) ; 128.19(\mathrm{C}-2,6) ; 133.00(\mathrm{C}-1) ; 136.78\left(\mathrm{PhCH}_{2}-1\right)$; $155.81(\mathrm{CO}) ; 157.26(\mathrm{C}-4) ; 161.39\left(\mathrm{C}-6^{\prime}\right) ; 165.38,165.56$ $\left(\mathrm{C}-2^{\prime}, 4^{\prime}\right) ; 170.91,171.46,204.36\left(\right.$ each CO). $\delta_{\mathrm{H}}(\mathrm{DMSO}-$ $\left.d_{6}\right): 1.70 \sim 4.46\left(\mathrm{~m}, \mathrm{OCH}_{2} \mathrm{CH}_{2} \mathrm{CHCONHCH}_{2}\right) ; 2.66 \sim 3.43$ $\left(4 \mathrm{H}, \mathrm{m}, \mathrm{PhCH}_{2} \mathrm{CH}_{2} \mathrm{CO}\right) ; 3.70,3.80\left(\right.$ each $\left.3 \mathrm{H}, \mathrm{s}, \mathrm{OCH}_{3}\right)$; $5.00\left(2 \mathrm{H}, \mathrm{s}, \mathrm{PhCH}_{2}\right) ; 6.05\left(2 \mathrm{H}, \mathrm{s}, \mathrm{H}-3^{\prime}, 5^{\prime}\right) ; 6.73(2 \mathrm{H}, \mathrm{d}, J=$ $8 \mathrm{~Hz}, \mathrm{H}-3,5) ; 7 . \overline{10}(2 \mathrm{H}, \mathrm{d}, J=8 \mathrm{~Hz}, \mathrm{H}-2,6) ; 7.30(5 \mathrm{H}, \mathrm{s}$, $\left.\mathrm{PhCH}_{2}\right) ; 7.60(1 \mathrm{H}, \mathrm{d}, J=8 \mathrm{~Hz}, \mathrm{NH}) ; 8.20$ (1 H, br.s, NH); $\overline{13} .40\left(1 \mathrm{H}, 6^{\prime}-\mathrm{OH}\right)$.

Hydrogenolysis of 23. A mixture of $23(500 \mathrm{mg})$ and $10 \%$ $\mathrm{Pd}-\mathrm{C}(400 \mathrm{mg})$ in dioxane $(15 \mathrm{ml})$ was stirred under hydrogen for $5 \mathrm{hr}$. The usual work-up gave 21 in an almost quantitative yield.

O-[(6'-Hydroxy $4,4^{\prime}$-dimethoxydihydrochalcone $)-2^{\prime}$ $y$ l Jhomoserine methyl ester hydrochloride (24). To a solution of $16(553 \mathrm{mg})$ in dioxane $(10 \mathrm{ml})$ were added $4.6 \mathrm{~N}$ hydrochloric acid in dioxane $(0.25 \mathrm{ml})$ and $10 \% \mathrm{Pd}-\mathrm{C}$ $(260 \mathrm{mg}$ ), before the mixture was stirred under hydrogen for $6 \mathrm{hr}$ at room temperature. To this, warm acetic acid was added to dissolve the reaction product deposited, and the mixture was filtered. The filtrate was evaporated, and the crystalline residue was washed with ether to yield $\mathbf{2 4}$ as plates $(370 \mathrm{mg}, 81.5 \%)$ mp $183 \sim 184^{\circ} \mathrm{C}$. Anal. Found: C, $58.23 ; \mathrm{H}, 6.07 ; \mathrm{N}, 3.00$. Calcd. for $\mathrm{C}_{22} \mathrm{H}_{27} \mathrm{O}_{7} \mathrm{~N} \cdot \mathrm{HCl}: \mathrm{C}$, $58.28 ; \mathrm{H}, 6.18 ; \mathrm{N}, 3.09 \%$. NMR $\delta_{\mathrm{C}}\left(\mathrm{CDCl}_{3}\right): 28.82\left(\mathrm{CH}_{2}\right.$ $\beta) ; 29.41\left(\mathrm{CH}_{2}\right) ; 45.16\left(\mathrm{CH}_{2} \alpha\right) ; 49.43\left(\mathrm{CH}_{3}\right) ; 52.80\left(\mathrm{CH}_{2}\right)$; $54.93,55.69\left(\right.$ each $\left.\left.\mathrm{OCH}_{3}\right) ; 64.61(\mathrm{CH}) ; 91.57(\mathrm{C}-3)^{\prime}\right) ; 94.11$ $\left(\mathrm{C}-5^{\prime}\right) ; 106.04\left(\mathrm{C}-1^{\prime}\right) ; 113.64(\mathrm{C}-3,5) ; 129.11(\mathrm{C}-2,6)$; 133.04 (C-1); 157.33 (C-4); 160.83 (C-6'); 165.02 (C-2'); $165.16\left(\mathrm{C}-4^{\prime}\right) ; 169.25,204.11$ (each $\left.\mathrm{CO}\right) . \delta_{\mathrm{H}}\left(\mathrm{CDCl}_{3}\right)$ : $2.10 \sim 4.42\left(\mathrm{~m}, \mathrm{OCH}_{2} \mathrm{CH}_{2} \mathrm{CH}, \mathrm{PhCH}_{2} \mathrm{CH}_{2} \mathrm{CO}, \mathrm{CHNH}\right.$, $\mathrm{PhOCH}_{2}$ ); 3.62, 3.65, $\overline{3.76}$ (each $\overline{3 \mathrm{H}}, \overline{\mathrm{s}}, 2 \mathrm{OCH}_{3}$ and $\left.\mathrm{CO}_{2} \overline{\mathrm{CH}}_{3}\right) ; 6.02\left(2 \mathrm{H}, \mathrm{s}, \mathrm{H}-3^{\prime}, 5^{\prime}\right) ; 6.70(2 \mathrm{H}, \mathrm{d}, J=8 \mathrm{~Hz}, \mathrm{H}-$ $3,5) ; 7.06(2 \mathrm{H}, \mathrm{d}, J=8 \mathrm{~Hz}, \mathrm{H}-2,6) ; 8.83$ (br.s, $3 \mathrm{H}$, $\left.\mathrm{NH}_{3}+\right) ; 13.32(1 \mathrm{H}, \mathrm{s}, \mathrm{OH})$.

$N-[($ Benzyloxycarbonyl $) g l y c y l]-O-\int\left(6^{\prime}\right.$-hydroxy-4,4'dimethoxydihydrochalcone)-2'-yl Jhomoserine methyl ester (25). Compound $24(1.81 \mathrm{~g})$ was added to DMF $(20 \mathrm{ml})$, and the suspension was stirred until a clear solution was obtained. After a while, long needles $\left(\mathbf{2 4}^{\prime}\right)$ were deposited from the solution. After filtration, $\mathbf{2 4}^{\prime}$ was washed with ether. This compound was an adduct of $\mathbf{2 4}$ and DMF, $\mathrm{mp}$ $179 \sim 180^{\circ} \mathrm{C}$. Anal. Found: C, $56.93 ; \mathrm{H}, 6.63 ; \mathrm{N}, 5.33$. Calcd. for $\mathrm{C}_{25} \mathrm{H}_{34} \mathrm{O}_{8} \mathrm{~N}_{2} \cdot \mathrm{HCl}$ : C, $57.03 ; \mathrm{H}, 6.65 ; \mathrm{N}, 5.32 \%$. NMR $\delta_{\mathrm{H}}\left(\mathrm{DMSO}-d_{6}\right): 2.00 \sim 4.40 \quad\left(\mathrm{~m}, \quad \mathrm{OCH}_{2} \mathrm{CH}_{2}\right.$, $\mathrm{PhCH}_{2} \mathrm{CH}_{2} \mathrm{CO}, \mathrm{CH}(\mathrm{NH}) \mathrm{CO}$ ); 2.73, 2.86 (each $3 \mathrm{H}, \mathrm{s}$, $\left.\mathrm{CH}_{3}\right) ; 3 . \overline{63}, 3.66,3.76\left(\right.$ each $\left.3 \mathrm{H}, \mathrm{s}, \mathrm{OCH}_{3}\right) ; 6.03(2 \mathrm{H}, \mathrm{s}, \mathrm{H}-$ $\left.3^{\prime}, 5^{\prime}\right) ; 6.73(2 \mathrm{H}, \mathrm{d}, J=8 \mathrm{~Hz}, \mathrm{H} 3,5) ; 7.06(2 \mathrm{H}, \mathrm{d}, J=8 \mathrm{~Hz}$, $\mathrm{H}-2,6) ; 7.83$ (1H, s, CHO); 8.73 (3H, br.s, $\mathrm{NH}_{3}+$ ). Compound $24^{\prime}$ was returned to the mother liquid, to which $N$-benzyloxycarbonylglycine $(803 \mathrm{mg})$, triethylamine $(1.2 \mathrm{ml})$ and DEPC $(0.64 \mathrm{ml})$ were added, before the clear solution was stood for $1 \mathrm{hr}$ at $0^{\circ} \mathrm{C}$ and then overnight at room temperature. The reaction mixture was worked up as described for the preparation of $\mathbf{1 3}$ from $\mathbf{8}$, and the final crystalline residue was recrystallized from ethanol to give 26 as needles $(1.65 \mathrm{~g}, 67.9 \%), \mathrm{mp} 124^{\circ} \mathrm{C}$. Anal. Found: $\mathrm{C}$, $63.01 ; \mathrm{H}, 5.88 ; \mathrm{N}, 4.89$. Calcd. for $\mathrm{C}_{32} \mathrm{H}_{36} \mathrm{~N}_{2} \mathrm{O}_{10}: \mathrm{C}, 63.16$; $\mathrm{H}, 5.92 ; \mathrm{N}, 4.61 \%$. NMR $\delta_{\mathrm{C}}\left(\mathrm{DMSO}-d_{6}\right): 28.80\left(\mathrm{CH}_{2} \beta\right)$; $30.30,43.38\left(\right.$ each $\left.\mathrm{CH}_{2}\right) ; 45.11\left(\mathrm{CH}_{2} \alpha\right) ; 48.85\left(\mathrm{CH}_{2}\right) ; 51.92$ $\left(\mathrm{CH}_{2}\right) ; 54.88,55.58\left(\right.$ each $\left.\mathrm{OCH}_{3}\right) ; 65.09\left(\mathrm{CH}_{2}\right) ; 65.44$ $(\mathrm{CH}) ; 91.39\left(\mathrm{C}-3^{\prime}\right) ; 93.80\left(\mathrm{C}-5^{\prime}\right) ; 105.61\left(\mathrm{C}-1^{\prime}\right) ; 113.56(\mathrm{C}-$ $3,5) ; 127.57,127.64\left(\mathrm{PhCH}_{2}-2 \sim 6\right) ; 128.21(\mathrm{C}-2,6) ; 132.97$ $(\mathrm{C}-1) ; 136.89\left(\mathrm{PhCH}_{2}-1\right) ; 156.34$ (CO); 157.29 (C-4); $\left.161.25\left(\mathrm{C}-6^{\prime}\right) ; 165.37\left(\mathrm{C}^{\prime}\right)^{\prime}\right) ; 165.61\left(\mathrm{C}^{\prime} 4^{\prime}\right) ; 169.31 ; 171.72$; 204.18 (each $\mathrm{CO}) . \delta_{\mathrm{H}}\left(\mathrm{DMSO}-d_{6}\right): 1.90 \sim 4.20 \quad(\mathrm{~m}$, $\mathrm{OCH}_{2} \mathrm{CH}_{2} \mathrm{CH}\left(\mathrm{NH}\right.$ ), $\mathrm{NHCH}_{2} \mathrm{CO}$ ); 3.50, 3.66, 4.03 (each $\left.3 \mathrm{H}, \mathrm{s}, \mathrm{OCH}_{3}\right) ; 4.90\left(2 \mathrm{H}, \mathrm{s}, \mathrm{PhCH}_{2}\right) ; 5.96(2 \mathrm{H}, \mathrm{s}, \mathrm{H}-3,5)$; $6.70\left(2 \mathrm{H}, \mathrm{d}, J=8 \mathrm{~Hz}, \mathrm{H}-3^{\prime}, 5^{\prime}\right) ; 7.06(2 \mathrm{H}, \mathrm{d}, J=8 \mathrm{~Hz}, \mathrm{H}-$ $\left.2^{\prime}, 6^{\prime}\right) ; 7.23\left(2 \mathrm{H}, \mathrm{s}, \mathrm{PhCH}_{2}\right) ; 8.23(1 \mathrm{H}, \mathrm{d}, J=8 \mathrm{~Hz}, \mathrm{NH})$; $13.40(1 \mathrm{H}, \mathrm{s}, \mathrm{OH})$.

$N-\int($ Benzyloxycarbonyl)glycyl $]-O-\int\left(6^{\prime}-h y d r o x y-4,4^{\prime}-\right.$ dimethoxydihydrochalcone)-2'-yl]homoserine (26). To a solution of $25(1.25 \mathrm{~g})$ in DMF $(16 \mathrm{ml})$ was added a $1 \mathrm{~N}$ sodium hydroxide solution $(2.6 \mathrm{ml})$, and the mixture was allowed to stand overnight at $23^{\circ} \mathrm{C}$. The reaction mixture 
was worked up in the same manner as described for the preparation of $\mathbf{2 3}$ from $\mathbf{2 2}$. The final residue was crystallized from ethanol to give 26 in a quantitative yield, $\mathrm{mp}$ $156^{\circ} \mathrm{C}$ (ethanol). Anal. Found: C, 62.49; H, 5.74; N, 4.74. Calcd. for $\mathrm{C}_{31} \mathrm{H}_{34} \mathrm{~N}_{2} \mathrm{O}_{10}: \mathrm{C}, 62.63 ; \mathrm{H}, 5.72 ; \mathrm{N}, 4.71 \%$. NMR $\delta_{\mathrm{C}}\left(\mathrm{DMSO}-d_{6}\right): 30.30\left(\mathrm{CH}_{2} \beta\right) ; 32.04,44.92$ (each $\left.\mathrm{CH}_{2}\right) ; 46.82\left(\mathrm{CH}_{2} \propto\right) ; 50.70\left(\mathrm{CH}_{2}\right) ; 55.54,56.01$ (each $\left.\mathrm{OCH}_{3}\right) ; 66.00(\mathrm{CH}) ; 68.32\left(\mathrm{CH}_{2}\right) ; 92.72\left(\mathrm{C}-3{ }^{\prime}\right) ; 94.73(\mathrm{C}-$ $\left.5^{\prime}\right) ; 106.68\left(\mathrm{C}-1^{\prime}\right) ; 114.75(\mathrm{C}-3,5) ; 128.79,129.00,129.35$, $130.21\left(\mathrm{PhCH}_{2}-2 \sim 6\right) ; 134.66(\mathrm{C}-1) ; 137.21\left(\mathrm{PhCH}_{2}-1\right)$; $158.75(\overline{\mathrm{CO}}, \mathrm{C}-4) ; 162.84\left(\mathrm{C}-6^{\prime}\right) ; 167.04\left(\mathrm{C}-2^{\prime}\right) ; \overline{16} 7.89(\mathrm{C}-$ $\left.4^{\prime}\right) ; 172.82,176.23,205.88$ (each $\left.\mathrm{CO}\right) . \delta_{\mathrm{H}}\left(\mathrm{CD}_{3} \mathrm{CO}_{2} \mathrm{D}\right)$ : $2.13 \sim 2.65\left(2 \mathrm{H}, \mathrm{m}, \mathrm{OCH}_{2} \mathrm{CH}_{2}\right) ; 2.65 \sim 3.53(4 \mathrm{H}, \mathrm{m}$, $\left.\mathrm{COCH}_{2} \mathrm{CH}_{2} \mathrm{Ph}\right) ; 3.70 \sim 4.30\left(\mathrm{OCH}_{2} \mathrm{CH}_{2}, \mathrm{NHCH}_{2} \mathrm{CO}\right)$; $3.66,3.69$ (each $\left.3 \mathrm{H}, \mathrm{s}, \mathrm{OCH}_{3}\right) ; 4.60 \sim 4.90(1 \overline{\mathrm{H}}$, br.s, $\mathrm{CHNH}) ; 5.03\left(2 \mathrm{H}, \mathrm{s}, \mathrm{PhCH}_{2}\right) ; 5.90,6.00$ (each s, d, $J=$ $\left.1.5 \mathrm{~Hz}, \mathrm{H}-3^{\prime}, 5^{\prime}\right) ; 6.70(2 \mathrm{H}, \overline{\mathrm{d}}, J=8 \mathrm{~Hz}, \mathrm{H}-3,5) ; 7.10(2 \mathrm{H}$, d, $J=8 \mathrm{~Hz}, \mathrm{H}-2,6) ; 7.21\left(5 \mathrm{H}, \mathrm{s}, \mathrm{PhCH}_{2}\right)$.

N-Glycyl-O-C(6'-hydroxy-4,4'-dimethoxydihydrochalcone)-2 -yl /homoserine (27). A mixture of $26(710 \mathrm{mg})$ and $10 \% \mathrm{Pd}-\mathrm{C}(480 \mathrm{mg})$ in acetic acid $(20 \mathrm{ml})$ was stirred under hydrogen for $6 \mathrm{hr}$ at room temperature. The reaction mixture was worked up as usual to give a syrup, which was crystallized from aqueous ethanol to give $\mathbf{2 7}$ as needles (407 mg, 69.9\%), mp $166 \sim 168^{\circ} \mathrm{C}$, decomposed above $200^{\circ} \mathrm{C}$. Anal. Found: C, 56.46; H, 5.98; N, 5.84\%. Calcd. for $\mathrm{C}_{23} \mathrm{H}_{28} \mathrm{~N}_{2} \mathrm{O}_{8} \cdot 1 \frac{1}{2} \mathrm{H}_{2} \mathrm{O}: \mathrm{C}, 56.67 ; \mathrm{H}, 6.36 ; \mathrm{N}, 5.75 \%$. NMR $\delta_{\mathrm{C}}\left(\mathrm{CD}_{3} \mathrm{CO}_{2} \mathrm{D}\right): 30.33\left(\mathrm{CH}_{2} \beta\right) ; 32.07,42.11$ (each $\left.\mathrm{CH}_{2}\right) ; 46.81\left(\mathrm{CH}_{2} \alpha\right) ; 51.17\left(\mathrm{CH}_{2}\right) ; 55.57,56.03$ (each $\left.\mathrm{OCH}_{3}\right) ; 65.94(\mathrm{CH}) ; 92.73\left(\mathrm{C}-3^{\prime}\right) ; 94.78\left(\mathrm{C}-5^{\prime}\right) ; 106.71(\mathrm{C}-$ $\left.1^{\prime}\right) ; 114.79(\mathrm{C}-3,5) ; 130.24(\mathrm{C}-2,6) ; 134.66(\mathrm{C}-1) ; 158.83$ $\left(\mathrm{C}-4^{\prime}\right) ; 162.40\left(\mathrm{C}^{\prime} 6^{\prime}\right) ; 162.83\left(\mathrm{C}-2^{\prime}\right) ; 167.10,168.29\left(\mathrm{C}-2^{\prime}\right.$, $\mathrm{CO}) ; 178.14,205.85$ (each $\mathrm{CO}) . \delta_{\mathrm{H}}\left(\mathrm{CD}_{3} \mathrm{CO}_{2} \mathrm{D}\right): 2.10 \sim$ $2.63\left(2 \mathrm{H}, \mathrm{m}, \mathrm{OCH}_{2} \mathrm{C}_{2}\right) ; 2.92,3.40($ each $2 \mathrm{H}, \mathrm{t}, J=6 \mathrm{~Hz}$, $\mathrm{COCH}_{2} \mathrm{CH}_{2} \mathrm{Ph}$ ); $3.63,3.76$ (each $\left.3 \mathrm{H}, 2 \mathrm{OCH}_{3}\right) ; 3.90 \sim 4.30$ $\left(4 \mathrm{H}, \overline{\mathrm{m}}, \overline{\mathrm{OCH}}_{2} \mathrm{CH}_{2}, \mathrm{NHCH}_{2} \mathrm{CO}\right) ; 4.66 \sim 4.90(1 \mathrm{H}, \mathrm{m}$, CHNH); 5.96, 6.10 (each $\left.1 \mathrm{H}, \mathrm{d}, J=2 \mathrm{~Hz}, \mathrm{H}-3^{\prime}, 5^{\prime}\right) ; 6.76$ $(2 \overline{\mathrm{H}}, \mathrm{d}, J=8 \mathrm{~Hz}, \mathrm{H}-3,5) ; 7.13(2 \mathrm{H}, \mathrm{d}, J=8 \mathrm{~Hz}, \mathrm{H}-2,6)$.

4,4',6'-Tribenzyloxy-2'-hydroxydihydrochalcone (29). To a solution of $2(3.97 \mathrm{~g})$ in DMF $(110 \mathrm{ml})$ were added benzyl bromide $(5.2 \mathrm{ml})$ and anhydrous potassium carbonate $(5.94 \mathrm{~g})$, before the mixture was stirred for $72 \mathrm{hr}$ at room temperature. The reaction mixture was poured into ice-cooled $1 \%$ hydrochloric acid with stirring, and the redish brown syrup deposited was extracted with ethyl acetate. The extract was washed with water, dried over $\mathrm{MgSO}_{4}$ and then evaporated. The residual syrup was chromatographed on a silica gel column, eluting with benzene-ethyl acetate $(9: 1)$, to give 29 , which was crystallized from ethanol $(1 \mathrm{~g}, 14.7 \%), \mathrm{mp} 105 \sim 106^{\circ} \mathrm{C}$. Anal. Found: $\mathrm{C}, 79.20 ; \mathrm{H}, 5.93$. Calcd. for $\mathrm{C}_{36} \mathrm{H}_{32} \mathrm{O}_{5}: \mathrm{C}, 79.41$; $\mathrm{H}, 5.88 \%$. Rf 0.90 (benzene-ethyl acetate, $9: 1$ ). NMR $\delta_{\mathrm{C}}$ $\left(\mathrm{CDCl}_{3}\right): 29.28\left(\mathrm{CH}_{2} \beta\right) ; 45.74\left(\mathrm{CH}_{2} \alpha\right) ; 69.94,70.11,71.16$ (each $\left.\mathrm{PhCH}_{2}\right) ; 92.23\left(\mathrm{C}-3^{\prime}\right) ; 94.89\left(\mathrm{C}-5^{\prime}\right) ; 105.95\left(\mathrm{C}-1^{\prime}\right)$; $114.64(\mathrm{C}-\overline{3}, 5) ; 127.26 \sim 128.60\left(\mathrm{PhCH}_{2}-2,6\right) ; 130.00(\mathrm{C}-$
$2,6) ; 133.55(\mathrm{C}-1) ; 135.27,135.81,137.24\left(\mathrm{PhCH}_{2}-1\right)$; $156.80(\mathrm{C}-4) ; 161.74\left(\mathrm{C}-6^{\prime}\right) ; 164.78\left(\mathrm{C}-2^{\prime}\right) ; 167.51\left(\mathrm{C}^{\prime} 4^{\prime}\right)$; $204.40(\mathrm{CO}) . \delta_{\mathrm{H}}\left(\mathrm{CDCl}_{3}\right): 2.66 \sim 2.96,2.96 \sim 3.36$ (each $\left.2 \mathrm{H}, \mathrm{m}, \mathrm{COCH}_{2} \mathrm{CH}_{2} \mathrm{Ph}\right) ; 4.93\left(6 \mathrm{H}, \mathrm{s}, 3 \mathrm{PhCH}_{2}\right) ; 5.96,6.06$ (each $\left.1 \mathrm{H}, \mathrm{d}, \vec{J}=1.5 \mathrm{~Hz}, \mathrm{H}-3^{\prime}, 5^{\prime}\right) ; 6.70(4 \mathrm{H}, \mathrm{s}, \mathrm{H}-2,6) ; 7.20$, $7.26(10 \mathrm{H}$, each s, $2 \mathrm{Ph}) ; 13.86(1 \mathrm{H}, \mathrm{s}, \mathrm{OH})$.

Further elution of the column gave a mixture of dibenzyloxy-2'-hydroxy-dihydrochalcones, which could not be separated, as well as other unidentified by-products.

$4,4^{\prime}, 6^{\prime}-$ Tribenzyloxy-2'-hydroxychalcone $\left(4,4^{\prime}, 6^{\prime}\right.$-tribenzyloxynaringeninchalcone, 31). A mixture of $\mathbf{3 0}(2.72 \mathrm{~g})$, benzyl bromide $(3.6 \mathrm{ml})$ and anhydrous potassium carbonate $(4.2 \mathrm{~g})$ in dry DMF $(70 \mathrm{ml})$ was stirred for $72 \mathrm{hr}$ at room temperature. The reaction mixture was poured into ice-cooled $1 \%$ hydrochloric acid $(500 \mathrm{ml})$, and a yellow precipitate $(5.5 \mathrm{~g})$ was collected by filtration, washed with water and dried in air. The well-dried sample was dissolved in benzene and chromatographed on a silica gel column, eluting with benzene-hexane $(7: 3)$ to give 31 , which was recrystallized from chloroform-ethanol as yellow plates. Yield, $2.68 \mathrm{~g}(56.1 \%), \mathrm{mp} 140^{\circ} \mathrm{C}$. Rf 0.61 (benzene). Anal. Found: C, 79.02; H, 5.53. Calcd. for $\mathrm{C}_{36} \mathrm{H}_{30} \mathrm{O}_{5}: \mathrm{C}, 79.70 ; \mathrm{H}, 5.54 \%$, NMR $\delta_{\mathrm{C}}\left(\mathrm{CDCl}_{3}\right): 70.03$, $70.23,71.37\left(\right.$ each $\left.\mathrm{CH}_{2}\right) ; 92.43\left(\mathrm{C}-3^{\prime}\right) ; 95.11\left(\mathrm{C}-5^{\prime}\right) ; 106.33$ $\left(\mathrm{C}-\mathrm{I}^{\prime}\right) ; 114.98(\mathrm{C}-3,5) ; 125.23(\mathrm{CH} \alpha) ; 127.37 \sim 128.85$ $\left(3 \mathrm{PhCH}_{2}-2 \sim 6\right) ; 130.07(\mathrm{C}-1) ; 135.46,135.90,136.54$ $\left(3 \overrightarrow{\mathrm{Ph}} \mathrm{CH}_{2}-1\right) ; 142.61(\mathrm{CH} \beta) ; 160.22\left(\mathrm{C}-6^{\prime}\right) ; 161.61(\mathrm{C}-4)$; $\left.165.00\left(\mathrm{C}^{\prime}\right)^{\prime}\right) ; 168.73\left(\mathrm{C}-4^{\prime}\right) ; 194.22(\mathrm{CO}) . \delta_{\underline{\mathrm{H}}}\left(\mathrm{CDCl}_{3}\right)$ : $5.00\left(6 \mathrm{H}, \mathrm{s}, 3 \mathrm{PhCH}_{2}\right) ; 6.10\left(2 \mathrm{H}, \mathrm{s}, \mathrm{H}-3^{\prime}, 5^{\prime}\right) ; 6.65(2 \mathrm{H}, \mathrm{d}$, $J=8 \mathrm{~Hz}, \mathrm{H}-3,5) ; 6.93(2 \mathrm{H}, \mathrm{d}, J=8 \mathrm{~Hz}, \mathrm{H}-2,6) ; 7.33(15 \mathrm{H}$, $\left.\mathrm{s}, 3 \mathrm{PhCH}_{2}\right) ; 7.66(2 \mathrm{H}, \mathrm{s}, \mathrm{H} \alpha, \beta) ; 14.60(1 \mathrm{H}, \mathrm{s}, \mathrm{OH})$.

$N$-Benzyloxycarbonyl-O-[ $\left[4,4^{\prime}, 6^{\prime}\right.$-tribenzyloxychalcone)-2'-yl l homoserine methyl ester (32). A mixture of 31 $(5.33 \mathrm{~g}), 15(3.26 \mathrm{~g})$ and anhydrous potassium carbonate $(1.4 \mathrm{~g})$ in dry acetone $(100 \mathrm{ml})$ was refluxed for $48 \mathrm{hr}$. After filtration, the filtrate was evaporated and the residue was chromatographed on a silica gel column, eluting with benzene-ethyl acetate (15:1) to afford $\mathbf{3 2}$ as a syrup $(5.7 \mathrm{~g}$, $73.2 \%$, which was contaminated with a minor amount of impurities. NMR $\delta_{\mathrm{C}}\left(\mathrm{CDCl}_{3}\right): 31.25,51.97\left(\right.$ each $\left.\mathrm{CH}_{2}\right)$; $52.29\left(\mathrm{CH}_{2}\right) ; 65.52(\mathrm{CH}) ; 66.67,70.02,70.28,70.46$ (each $\left.\mathrm{PhCH}_{2}\right) ; 93.36,93.70\left(\mathrm{C}-3^{\prime}, 5^{\prime}\right) ; 113.27\left(\mathrm{C}-1^{\prime}\right) ; 115.10(\mathrm{C}-$ $3,5) ; 126.96 \sim 130.02\left(\mathrm{CH} \alpha, 4 \mathrm{PhCH}_{2}-2 \sim 6\right) ; 136.39$, $\left.136.46,136.55\left(4 \mathrm{PhCH}_{2}-1\right) ; 143.85 \overline{(\mathrm{CH} \beta}\right) ; 157.80,157.90$ $\left(\mathrm{C}-4,6^{\prime}\right) ; 160.43 \overline{\left(\mathrm{C}-2^{\prime}\right) ; 161.32\left(\mathrm{C}^{\prime} 4^{\prime}\right) ; 171.92,193.22(\mathrm{each}}$ $\mathrm{CO})$.

$N$-Benzyloxycarbonyl-O- $\left[4,4^{\prime}, 6^{\prime}\right.$-tribenzyloxychalcone)$2^{\prime}$-yl Jhomoserine (33). To a suspension of crude $32(5.7 \mathrm{~g})$ in $4 \%$ sodium hydroxide solution $(20 \mathrm{ml})$ was added acetone until a clear solution was obtained. After standing for $2 \mathrm{hr}$ at room temperature, the solution was neutralized with acetic acid and evaporated. The residue was dissolved in a small quantity of DMF and poured into ice-cooled $1 \%$ hydrochloric acid, and the precipitate was extracted with 
ethyl acetate. The extract was washed with brine, dried over $\mathrm{MgSO}_{4}$, and evaporated. The residue was crystallized from ethanol to give $\mathbf{3 3}$ as needles $(2.94 \mathrm{~g}, 52.5 \%$, mp $123.5^{\circ} \mathrm{C}$. Anal. Found: C, 73.93; H, 5.56; N, 1.73. Calcd. for $\mathrm{C}_{48} \mathrm{H}_{43} \mathrm{NO}_{9}: \mathrm{C}, 74.13 ; \mathrm{H}, 5.53 ; \mathrm{N}, 1.80 \%$. NMR $\delta_{\mathrm{C}}$ $\left(\mathrm{CDCl}_{3}\right)$ : 31.20, 51.91 (each $\left.\mathrm{CH}_{2}\right) ; 65.00(\mathrm{CH}) ; 66.83$, $70.28,70.44,70.58\left(4 \mathrm{PhCH}_{2}\right) ; 93.07,93.54\left(\mathrm{C}-3^{\prime}, 5^{\prime}\right)$; $112.47 \quad\left(\mathrm{C}^{-1}\right) ; \quad 115.09 \quad(\mathrm{C}-3,5) ; 126.94 \quad(\mathrm{CH} \quad \alpha)$; $127.35 \sim 130.19 \quad\left(4 \mathrm{PhCH}_{2}-2 \sim 6\right) ; 136.28 \quad\left(4 \mathrm{PhCH}_{2}-1\right)$; $144.73(\mathrm{CH} \quad \beta) ; 1 \overline{56.10}\left(\mathrm{C}-6^{\prime}\right) ; 157.84,15 \overline{8.00}(\mathrm{C}-4$,

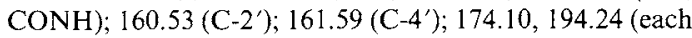
CO). $\delta_{\mathrm{H}}\left(\mathrm{CDCl}_{3}\right): 2.13\left(2 \mathrm{H}\right.$, br. s, $\left.\mathrm{OCH}_{2} \mathrm{CH}_{2}\right) ; 3.70 \sim 4.00$ $\left(2 \mathrm{H}, \mathrm{m}, \mathrm{OH}_{2} \mathrm{CH}_{2}\right) ; 4.20 \sim 4.50(1 \mathrm{H}, \mathrm{m}, \mathrm{CH} \overline{\mathrm{NH}}) ; 4.83,4.86$, $4.90,4.93\left(\right.$ each $\left.2 \mathrm{H}, \mathrm{s}, \mathrm{PhCH}_{2}\right) ; 5.83(1 \mathrm{H}, \mathrm{d}, J=6 \mathrm{~Hz}, \mathrm{NH})$; $6.08\left(2 \mathrm{H}, \mathrm{d}, J=3 \mathrm{~Hz}, \mathrm{H}-3^{\prime}, 5^{\prime}\right) ; 6.70(2 \mathrm{H}, \mathrm{d}, J=8 \mathrm{~Hz}, \mathrm{H}-$ $3,5) ; 6.75(2 \mathrm{H}, \mathrm{d}, J=8 \mathrm{~Hz}, \mathrm{H}-2,6) ; 7.03,7.13,7.20(22 \mathrm{H}$, each $\mathrm{s}, \mathrm{CH}=\mathrm{CH}, \underline{4 \mathrm{PhCH}_{2}}$ ).

[N-Benzyloxycarbonyl-O-[ $\left[4,4^{\prime}, 6^{\prime}\right.$-tribenzyloxychalcone)-2'-yl Jhomoseryl Jglycine benzyl ester (34). A mixture of $33(2.94 \mathrm{~g}), 19(1.3 \mathrm{~g})$, triethylamine $(1.1 \mathrm{ml})$ and DEPC $(0.65 \mathrm{ml})$ in DMF $(24 \mathrm{ml})$ was stirred for $1 \mathrm{hr}$ at $0^{\circ} \mathrm{C}$ and then overnight at room temperature. The reaction mixture was worked-up as described before, and the final syrup was crystallized from chloroform-ethanol to give $\mathbf{3 4}$ as needles $\left(3 \mathrm{~g}, 85.8 \%\right.$ ), mp $118^{\circ} \mathrm{C}$ (chloroform-ethanol). Anal. Found: C, 73.78; H, 5.61; N, 2.96. Calcd. for $\mathrm{C}_{57} \mathrm{H}_{52} \mathrm{~N}_{2} \mathrm{O}_{10}: \mathrm{C}, 74.03 ; \mathrm{H}, 5.63 ; \mathrm{N}, 3.03 \%$. NMR $\delta_{\mathrm{C}}$ $\left(\mathrm{CDCl}_{3}\right): 32.23,41.30,52.93\left(\right.$ each $\left.\mathrm{CH}_{2}\right) ; 65.64(\mathrm{CH})$; $66.69,70.26,70.41 \quad\left(5 \mathrm{PhCH}_{2}\right) ; 93.12,93.44\left(\mathrm{C}-3^{\prime}, 5^{\prime}\right)$; $112.61\left(\mathrm{C}-1^{\prime}\right) ; 115.09(\mathrm{C}-3,5) ; 126.78(\mathrm{CH} \alpha) ; 126.95 \sim$ $130.16\left(5 \mathrm{PhCH}_{2}-2 \sim 6\right) ; \quad 136.17 \sim 136.46 \quad\left(5 \mathrm{PhCH}_{2}-1\right)$; $144.01(\mathrm{CH} \beta) ; 155.90$ (C-6'); 158.08 (C-4, CONH); $160.49\left(\mathrm{C}-2^{\prime}\right) ; 161.65\left(\mathrm{C}-4^{\prime}\right) ; 168.99(\mathrm{CONH}) ; 171.68$, $193.74($ each $\mathrm{CO}) . \delta_{\mathrm{H}}\left(\mathrm{CDCl}_{3}\right): 1.80 \sim 2.50(2 \mathrm{H}, \mathrm{m}$, $\left.\mathrm{OCH}_{2} \mathrm{CH}_{2}\right) ; 3.76 \sim 4.50\left(5 \mathrm{H}, \mathrm{m}, \mathrm{OCH}_{2} \mathrm{CH}_{2}, \mathrm{CH}(\mathrm{NH})\right.$, $\left.\mathrm{NHCH}_{2} \mathrm{CO}\right) ; 4.80 \sim 5.40\left(10 \mathrm{H}, \mathrm{m}, 5 \mathrm{PhCH}_{2}\right) ; 6.20(2 \mathrm{H}, \mathrm{s}$, $\left.\mathrm{H}-3^{\prime}, \overline{5^{\prime}}\right) ; 6.80(2 \mathrm{H}, \mathrm{d}, J=9 \mathrm{~Hz}, \mathrm{H}-3,5) ; 7.00(2 \mathrm{H}, \mathrm{d}, J=$ $9 \mathrm{~Hz}, \mathrm{H}-2,6) ; 7.00 \sim 8.20\left(27 \mathrm{H}, \mathrm{m}, 5 \mathrm{PhCH}_{2}, \mathrm{CH}=\mathrm{CH}\right)$. UV. $i_{\max }(\mathrm{nm}): 330$.

[O- $\left[\left(4,4^{\prime}, 6^{\prime}-\right.\right.$ Trihydroxydihydrochalcone $\left.)-2^{\prime}-y /\right]$ homosery Jglycine hydrochloride (28). A mixture of $\mathbf{3 4}$ (924 mg) and $10 \% \mathrm{Pd}-\mathrm{C}(900 \mathrm{mg})$ in dioxane $(70 \mathrm{ml})$ containing conc. hydrochloric acid $(0.5 \mathrm{ml})$ was stirred under hy- drogen for $48 \mathrm{hr}$ at room temperature. The reaction mixture was worked up as usual to give a very hygroscopic powder $(\mathbf{2 8} ; 420 \mathrm{mg}, 87.3 \%)$. The ninhydrin test was positive. NMR $\delta_{\mathrm{C}}\left(\mathrm{DMSO}-d_{6}\right): 29.03\left(\mathrm{CH}_{2} \beta\right) ; 30.33,44.99$ (each $\left.\mathrm{CH}_{2}\right) ; 49.65\left(\mathrm{CH}_{2} \alpha\right) ; 62.77\left(\mathrm{CH}_{2}\right) ; 64.3 \mathrm{I}(\mathrm{CH}) ; 92.08$ $\left(\mathrm{C}-3^{\prime}\right) ; 95.91\left(\mathrm{C}^{\prime} 5^{\prime}\right) ; 104.79\left(\mathrm{C}-1^{\prime}\right) ; 115.01(\mathrm{C}-3,5) ; 128.95$ $(\mathrm{C}-2,6) ; 131.21$ (C-1); 155.24 (C-4); $161.50\left(\mathrm{C}^{\prime}\right) ; 164.50$

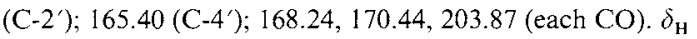
$\left(\mathrm{CDCl}_{3}\right): 5.93\left(2 \mathrm{H}, \mathrm{s}, \mathrm{H}-3^{\prime}, 5^{\prime}\right) ; 6.60(2 \mathrm{H}, \mathrm{d}, J=9 \mathrm{~Hz}, \mathrm{H}-$ $3,5) ; 6.90(2 \mathrm{H}, \mathrm{d}, J=9 \mathrm{~Hz}, \mathrm{H}-2,6) ; 8.85\left(3 \mathrm{H}\right.$, br. s, $\left.\mathrm{NH}_{3}{ }^{+}\right)$; $13.21(1 \mathrm{H}, \mathrm{s}, \mathrm{COOH})$. UV $i_{\max }(\mathrm{nm}): 283,222$.

In the abscence of hydrochloric acid or in the presence of acetic acid instead of hydrochloric acid, the reaction products showed negative to the ninhydrin test. In the ${ }^{1} \mathrm{H}$ NMR spectrum, the signals of the $\mathrm{NH}_{3}{ }^{*}$ and $\mathrm{COOH}$ groups were not recognized. It is thought that the diketopiperazine ring was perhaps formed in $\mathbf{2 8}$.

\section{References}

1) de L. Koninck, Liebigs Ann. Chem., 15, 75, 258 (1835); J. S. Challica and A. H. Williams, Phytochemistry, 9, 1261 (1970)

2) D. F. Diedrich, Ark. Biochem. Biophys., 117, 248 (1966); D. F. Diedrich, Biochim. Biophys. Acta, 71, 688 (1966).

3) E. Brot-Laroche and F. Alvarado, in "Intestinal Transport," ed. by M. Gilles-Baillien and R. Gilles, Springer, Berlin, 1983, pp. $147 \sim 169$.

4) F. Alvarado, Biochim. Biophys. Acta, 135, 483 (1967).

5) T. Hoshi, N. Takuwa, M. Abe and A. Tajima, Biochim. Biophys. Acta, 861, 483 (1986).

6) M. Abe, T. Hoshi and A. Tajima, J. Physiol. (London), 394, 481 (1987).

7) T. Hoshi, Jpn. J. Physiol., 35, 179 (1985).

8) Y. Asahina and M. Inubuse, Yakugaku Zasshi, 48, 868 (1928). E. Wollenweber and K. Egger, Ztsch. Pflanzenphysiol., 65, 427 (1971).

9) W. Klyne, Biochem. J., 47, XLI (1950).

10) S. Takuma, Y. Hamada and T. Shioiri, Chem. Pharm. Bull., 38, 3147 (1982).

11) G. D. DuBois, G. A. Crosby, J. F. Lee, R. A. Stephenson and P. C. Wang, J. Agric. Food Chem., 29, 1261 (1981). 